\title{
Caspase-dependent cleavage of DDX21 suppresses host innate
}

\section{immunity}

3 Wei $\mathrm{Wu}^{1}$, Yang $\mathrm{Qu}^{2}$, Shengqing $\mathrm{Yu}^{1}$, Sa Wang${ }^{3}$, Yuncong $\mathrm{Yin}^{4}$, Qinfang $\mathrm{Liu}^{1}$,

4 Chunchun Meng ${ }^{1}$, Ying Liao', Zaib Ur Rehman'스, Lei Tan', Cuiping Song ${ }^{1}$, Xusheng

5 Qiu ${ }^{1}$, Weiwei Liu ${ }^{1}$, Chan Ding ${ }^{1,4^{*}}$, Yingjie Sun ${ }^{1 *}$

6 Running head: DDX21 cleavage suppresses innate immunity

81 Department of Avian Infectious Diseases, Shanghai Veterinary Research Institute,

9 Chinese Academy of Agricultural Science, Shanghai, 200241, P.R. China

2 College of Veterinary Medicine, Northwest A\&F University, Yangling, 712100,

11 Shaanxi, PR China

123 College of Animal Sciences, Fujian Agriculture and Forestry University, Fuzhou

13 350002, P.R. China

144 Jiangsu Co-innovation Center for Prevention and Control of Important Animal

15 Infectious Diseases and Zoonoses, Yangzhou, 225009, P.R. China

$16 *$ Equal corresponding Author:

17 Address: 518 Ziyue Road, Shanghai 200241, P. R. China

18 Phone: $+86-21-34293441$

19 Fax: +86-21-54081818

20 E-mail address: sunyingjie@ shvri.ac.cn; shoveldeen@ shvri.ac.cn 
21 Abstract: DEAD (Glu-Asp-Ala-Glu)-box RNA helicases have been proven to

22 contribute to antiviral innate immunity. DDX21 RNA helicase was identified as a

23 nuclear protein involved in ribosomal RNA processing and RNA unwinding. DDX21

24 was also proved to be the scaffold protein in the complex of DDX1-DDX21-DHX36

25 which senses double strand RNA and initiates downstream innate immunity. Here, we

26 identified that DDX21 undergoes caspase-dependent cleavage after virus infection

27 and treatment with RNA/DNA ligands, especially for RNA virus and ligands.

28 Caspase-3/6 cleave DDX21 at $\mathrm{D}^{126}$ and promotes its translocation from the nucleus to

29 the cytoplasm in response to virus infection. The cytoplasmic cleaved DDX21

30 negatively regulates the IFN- $\beta$ signaling pathway by suppressing the formation of

31 DDX1-DDX21-DHX36 complex. Thus, our data identify DDX21 as a regulator of

32 immune balance and most importantly uncover a potential role of DDX21 cleavage in

33 the innate immunity response towards virus.

35 Keywords: DDX21, virus infection, cleavage, innate immunity 


\section{Importance}

37 Innate immunity serves as the first barrier against virus infection. DEAD

38 (Glu-Asp-Ala-Glu)-box RNA helicases, originally considered to be involved RNA

39 processing and RNA unwinding, have been shown to play an important role in

40 anti-viral innate immunity. The precise regulation of innate immunity is critical for the

41 host because the aberrant production of cytokines leads to unexpected pathological

42 consequences. Here, we identified DDX21 was cleaved at $\mathrm{D}^{126}$ by virus infection and treatment with RNA/DNA ligands via the caspase-3/6-dependent pathway. The cytoplasmic cleaved DDX21 negatively regulates the IFN- $\beta$ signaling pathway by suppressing the formation of DDX1-DDX21-DHX36 complex. In sum, our data identify DDX21 as a regulator of immune balance and most importantly uncover a potential role of DDX21 cleavage in the innate immunity response towards virus. 


\section{Introduction}

The host innate immunity response is initiated by virus infection. Several pathogen recognition receptors (PRRs) were mobilized to sense viral nucleic acids and ultimately lead to the induction of interferons (IFNs) and other inflammatory cytokines to protect host cells (1). Among them, endosomal toll-like receptor 3 (TLR3) (2, 3), cytoplasmic retinoic acid-inducible gene I (RIG-I), and melanoma differentiation-associated protein 5 (MDA5) $(4,5)$ were demonstrated to be critical for sensing viral double-stranded RNA (dsRNA). The adaptor protein TIR domain-containing adaptor inducing interferon- $\beta$ (TRIF) (2) and mitochondrial protein MAVS (VISA, IPS-1, or Cardif) (6-9) then activated, leading to the activation of nuclear factor kappa $\mathrm{B}(\mathrm{NF}-\mathrm{\kappa B})$ and transfection factor interferon regulatory factor 3 (IRF3) as well as the production of various cytokines, including type I-IFNs (10). The secreted IFN binds to the IFN receptor and induces the expression of various interferon-stimulated genes (ISGs) to establish a cellular anti-viral state (1). The helicase family is a class of enzymes that are essential to all living organisms. Their main function is separating nucleic acid strands (DNA, RNA, or RNA:DNA hybrid) (11). The human genome encodes 64 RNA helicases and 31 DNA helicases, which are classified in several superfamilies (SFs) based on their conserved motifs $(12,13)$. DEAD/H box helicase belongs to the SF2 family, the largest group of helicases, which are involved in various cellular processes $(12,13)$. RNA helicases are critical for most RNA metabolism processes and are also involved in the antiviral 
70 immune response by sensing foreign RNAs (14). RIG-I, MDA5, and laboratory of

71 genetics and physiology 2 (LGP2), three RIG-I-like receptors (RLRs) that belong to

72 SF2 RNA helicases, are closely related to DEAD-box helicases (15). In addition to

73 RLRs, a growing list of DEAD/H box helicases has been identified to contribute to

74 antiviral innate immunity in recent years, either by acting as sensors for viral nucleic

75 acids or as mediators of downstream signaling events (16-21).

DDX21 was shown to be an abundant nuclear protein in HeLa cells that directly

binds rRNAs and snoRNAs and promotes rRNA transcription, processing, and

modification (22-25). Another important function of DDX21 is unwinding RNAs,

including dsRNA and RNA guanine quadruplexes (26-28). Recently, several reports indicated that DDX21 also plays a role in innate immunity and virus infection.

81 DDX21, together with DDX1 and DHX36, can bind the adaptor protein TRIF to sense dsRNA (20). During dengue virus infection, DDX21 translocates from nucleus to cytoplasm and mediates innate immunity (29). Moreover, DDX21 inhibits influenza A virus replication but is counteracted by viral NS1 protein (30). However, the way in which DDX21 precisely regulates anti-viral innate immunity and whether DDX21 undergoes protein modification during virus infection remain unclear. manipulates the expression of several DDX/DHXs during infection (data not shown). Among them, DDX21 was found to be cleaved in HeLa cells infected with NDV. 
91 the caspase-3/6 pathway. The cleavage of DDX21 promotes its translocation from the

92 nucleus to the cytoplasm in response to virus infection. The cytoplasmic cleaved

93 DDX21 negatively regulates the IFN- $\beta$ signaling pathway by suppressing the

94 formation of DDX1-DDX21-DHX36 complex. Our study therefore reveals a role of

95 DDX21 in the regulation of anti-viral innate immunity and provides molecular

96 insights into how the host balances anti-viral and aberrant innate immunity. 


\section{Materials and Methods}

\section{Reagents and antibodies}

Caspase inhibitor z-VAD-FMK (C1202, Beyotime, Nantong, China) was used at a concentration of $50 \mu \mathrm{M}$. Neddylation inhibitor MLN4924 (S7109, Selleckchem, Houston, TX, USA) was used at a concentration of $1 \mu \mathrm{M}$. Proteasome inhibitor MG-132 (S1748, Beyotime) was used at a concentration of $20 \mu \mathrm{M}$. The autophagy inhibitors wortmannin (W1628, Sigma-Aldrich, St. Louis, MO, USA) and chloroquine (CQ) (C6628, Sigma-Aldrich) were used at $300 \mathrm{nM}$ and $25 \mu \mathrm{M}$ concentrations, respectively. Poly(I:C) (tlrl-pic), 3p-hpRNA (tlrl-hprna), HSV-60 (tlrl-hsv60n) and poly(dG:dC) (tlrl-pgen) were purchased from Invivogen (San Diego, CA, USA). Rabbit monoclonal anti-DDX21 (ab182156), anti-caspase-6 (ab185645), mouse monoclonal anti-VSV-glycoprotein (G) antibodies (ab50549), and rabbit polyclonal anti-Lamin B1 antibodies (ab16048) were purchased from Abcam (Cambridge, MA, USA). Rabbit polyclonal anti-HSV-1-glycoprotein D (gD) (NB600-516) was purchased from Novus biologicals (Littleton, CO). Rabbit polyclonal anti-Sendai (PD029C1) was purchased from MBL (Nagoya, Japan). Rabbit polyclonal anti-caspase-3 (GTX110543) was purchased from GeneTex (Irvine, CA).

Mouse monoclonal anti-Flag (F1804), anti-HA (H9658), and anti- $\beta$-actin antibody (A1978) were purchased from Sigma-Aldrich. Rabbit monoclonal anti-phosphoTANK Binding Kinase 1 (TBK1) (\#5483) and anti-TBK1 (\#3013) were purchased from Cell Signaling Technology (Beverly, MA, USA). Monoclonal antibody against 
118 NDV nucleoprotein (NP) was prepared in our laboratory (31). For the

119 immunofluorescence assays, mouse monoclonal anti-HSV-1/2 $\mathrm{gE}$ was purchased

120 from Santa Cruz Biotechnology (Dallas, TX, USA), and mouse polyclonal

121 anti-VSV-G antibodies (ab1874) were purchased from Abcam. IFN- $\beta$ was measured

122 with an ELISA kit (41410, PBL Assay Science, Piscataway, NJ).

\section{Cell cultures and virus}

124 HeLa, A549, HEK-293T, Vero, HuH7, and THP-1 cells were purchased from

125 American Type Culture Collection (ATCC). These cells were maintained in

126 Dulbecco's modified Eagle's medium (DMEM) supplemented with $10 \%$ fetal bovine

127 serum (Thermo Fisher Scientific, Waltham, MA, USA). NDV Herts/33 strain was

128 obtained from the China Institute of Veterinary Drug Control (Beijing, China). Herpes

129 simplex virus (HSV)-1 was kindly provided by Yasushi Kawaguchi (University of

130 Tokyo, Japan), and vesicular stomatitis virus (VSV) was provided by Jianchao Wei

131 (Shanghai Veterinary Research Institute, China). Sendai virus (SeV) was provided by

132 Quan Zhang (Yangzhou University, China). HSV-1 and VSV titration was determined

133 as the median tissue culture infective dose $\left(\mathrm{TCID}_{50}\right)$ on Vero cells.

\section{Plasmids}

135 Flag-tagged DDX21-X1 (Flag-DDX21) and DDX21-X2 were constructed by

136 inserting the open reading frame (ORF) of human DDX21 isoform 1 (NM_004728.4)

137 and isoform 2 (NM_001256910.1) into plasmid p3XFLAG-CMV-14 (Sigma-Aldrich), 
138 respectively. Flag-tagged deletion constructs $(\Delta 217-395, \Delta 396-573)$ and point mutants

139 of DDX21 (D87A, D126A, 160A, D87A/D126A, D87A/160A, D126A/D160A,

140 D81A/D126A/160A) were generated by site-directed mutagenesis, as described

141 previously $(32,33)$. Flag- and HA-tagged wide-type and truncates of DDX21 (1-125,

$142127-784, \Delta 1-216, \Delta 574-784)$ were constructed by inserting indicated sequences into

143 p3XFLAG-CMV-14 (Sigma-Aldrich) and pCMV-HA (Promega), respectively.

144 HA-tagged DDX1and HA-tagged TRIF were constructed by inserting indicated

145 sequences into pCMV-HA (Promega), respectively. Myc-tagged DHX36 was

146 constructed by inserting the ORF of DHX36 into pCMV-Myc (Promega).

147 pHAGE-WT and -D126A DDX21 were constructed by inserting the flag-tagged WT

148 and D126A DDX21 into pHAGE-bsd, which was constructed based on pHAGE-puro

149 (Addgene, plasmid 118692). The primer sequences for plasmid construction are listed

150 in Supplementary Table 1 . The IFN- $\beta$ promoter luciferase reporter was kindly

151 provided by Takeshi Fujita (Kyoto University, Japan).

\section{Cell transfection, luciferase assay, and gene knockdown}

153 Cells were transfected using FuGENE® HD (Promega, Madison, WI, USA) or

154 Lipofectamine ${ }^{\circledR} 2000$ (Thermo Fisher Scientific) according to the manufacturer's

155 instructions. For the luciferase assay, cells were cultured in 24-well plates and

156 co-transfected with $100 \mathrm{ng}$ of firefly luciferase reporter (IFN- $\beta$-Luc) and $10 \mathrm{ng}$ of the

157 constitutive Renilla luciferase reporter pRL-TK. Luciferase activity was measured at

$15824 \mathrm{~h}$ post transfection (hpt). Lentiviral shRNA for targeting endogenous DDX21 
159 (5'-CCCATATCTGAAGAAACTATT-5') were purchased from Gene Pharma

160 (Shanghai, China). To generate DDX21 stable knockdown cell line, HeLa cells were

161 infected with lentiviral shDDX21 and selected by puromycin as described previously

\section{Immunofluorescence (IF) assay}

164 HeLa cells were washed in PBS, fixed in $4 \%$ neutral formaldehyde, and then

165 permeabilized with $0.5 \%$ Triton X-100 in Tris-buffered saline with Tween 20 (TBST)

166 for $10 \mathrm{~min}$. After blocking in TBST with $3 \%$ bovine serum albumin, the cells were

167 incubated with primary antibody for $1 \mathrm{~h}$ at $37^{\circ} \mathrm{C}$. The cells were washed three times

168 with TBST and incubated with secondary antibody. Subsequently, they were washed

169 and incubated with another primary antibody, followed by incubation with secondary

170 antibody. Next, the cells were washed again and incubated with $0.5 \mu \mathrm{g} / \mathrm{mL}$ DAPI. The

171 coverslips were washed and visualized using an LSM 880 Zeiss confocal microscope

172 (Carl Zeiss, Jena, Germany).

173 Nucleocytoplasmic separation assay

174 Nuclear extractions were prepared using NE-PER nuclear and cytoplasmic extraction

175 reagents (78833, ThermoFisher Scientific) according to the manufacturer's

176 instructions. 


\section{Immunoblotting and co-immunoprecipitation}

178 Immunoblotting was performed as described previously (31). Briefly, cells were lysed

179 in cell lysis buffer containing a protease inhibitor cocktail (Merck Millipore,

180 Darmstadt, Germany). The lysates were denatured and then subjected to sodium

181 dodecyl sulfate polyacrylamide gel electrophoresis (SDS-PAGE) and immunoblotting

182 and quantified using Image $\mathbf{J}$ software. For co-immunoprecipitation, HeLa cells were

183 transfected with expression vectors for 24 or $36 \mathrm{~h}$ and lysed with a cell lysis buffer

184 (150 mM NaCl, 50 mM Tris-HCl, pH 8.0, 5 mM EDTA, 0.5\% NP-40) containing 1

$185 \mathrm{mM}$ PMSF and protease inhibitors (Merck-Millipore). The lysates were centrifuged at

$18612,000 \mathrm{~g}$ for $10 \mathrm{~min}$ and precipitated with anti-Flag antibody, in conjunction with

187 Protein G Agarose beads (ThermoFisher Scientific) overnight at $4{ }^{\circ} \mathrm{C}$. The beads were

188 washed with lysis buffer four times and eluted with SDS loading buffer by boiling for

$18910 \mathrm{~min}$ and then subjected to immunoblotting.

190 Quantitative real-time PCR

191 Quantitative real-time PCR (qRT-PCR) was performed as described previously (35).

192 Briefly, total RNA was extracted, reverse transcribed to cDNA, and subjected to

193 qRT-PCR analysis using Premix Ex Taq reagents (Takara, Dalian, China). Primers

194 were referenced from previous reports (35) (Supplementary Table 1). The relative

195 abundance of mRNAs was calculated using the comparative $\mathrm{C}_{\mathrm{T}}(\Delta \Delta \mathrm{CT})$ method (36).

196 All experiments were carried out in triplicate. 


\section{Generation of knockout cells}

198 The CRISPR/Cas9 system was used to generate $d d x 21$ and casp6 knockout cells. The 199 guide RNA (gRNA) specific to $d d x 21$ and casp6 gene was designed using the online

200 CRISPR design tool (http://crispr.mit.edu/). The gRNA oligonucleotides were

201 annealed and cloned into the pGK1.1 (Geneloci, Nanjing, China). HeLa cells were

202 electrotransfected with the plasmid at 550V, 1 pulse. After 24 h of electrotransfection,

203 the supernatants were replaced with $10 \%$ FBS DMEM supplemented with $1 \mu \mathrm{g} / \mathrm{ml}$

204 puromycin (Merck-Millipore) for $24 \mathrm{~h}$. The pool (mixed clones) cells were

205 preliminarily sequenced and validated. The candidate positive clones were then

206 subcloned onto 96-well plates using the limiting dilution method. The genomic region

207 surrounding the CRISPR target site was amplified by PCR using the check primers.

208 The primer sequences for sgRNAs and check primers are listed in Supplementary

209 Table 1. The clones were sequenced to ensure the frameshifting mutation of both

210 alleles of the established cell line. Except for PCR verification, the cells were

211 re-checked by immunoblotting using DDX21 antibody. Casp3-/- HeLa cells were

212 purchased from EdiGene Inc. (Beijing, China) and verified by immunoblotting.

213 Casp3/6 double knockout cells were constructed by transfecting casp6 sgRNA into

214 casp3-/- HeLa cells as described above.

215 Generation of cells stably expressing WT and mutant DDX21

216 HEK-293T cells were transfected with pHAGE-WT and -D126A DDX21,

217 together with two package plasmids, pMD2.G (Addgene, plasmid 12259) and 
218 psPAX2 (Addgene, plasmid 12260). The supernatants were collected at $60 \mathrm{~h}$ post

219 transfection (hpt), centrifuged at $5000 \mathrm{rpm}$ for $10 \mathrm{~min}$, and filtered. The lentivirus

220 supernatants supplemented with $5 \mu \mathrm{g} / \mathrm{mL}$ polybrene (Sigma-Aldrich) were added into

221 the $d d x 21+/-$ HeLa cells. The cells were horizontally centrifuged at $1000 \mathrm{rpm}$ for 90

$222 \min$ and incubated at $37^{\circ} \mathrm{C}$ for $48 \mathrm{~h}$. The supernatants were then replaced with $10 \%$

223 FBS supplemented with blasticidin (Merck-Millipore) for $72 \mathrm{~h}$. The cells were

224 subcloned by limiting dilution and confirmed by immunoblotting. The primer

225 sequences for stable expression are listed in Supplementary Table 1.

\section{Statistical analysis}

227 Data were expressed as means \pm standard deviations. Significance was determined

228 with the two-tailed independent Student's $t$ test $(p<0.05)$ between two groups. A

229 one-way ANOVA was followed by Tukey’s to compare multiple groups $(>2)$. 


\section{Results}

\section{DDX21 positively regulates IFN- $\beta$ signaling pathway}

233 To confirm the role of DDX21 in virus replication as well as its role in anti-viral

234 innate immunity, DDX21 was knocked down followed by VSV infection. The results

235 showed that the knockdown of DDX21 significantly reduced the expression of VSV

$236 \mathrm{G}$ protein at 12, 18, and $24 \mathrm{~h}$ post infection (hpi) (Fig. 1A). Consistently, the virus

237 titers were significantly impaired after DDX21 knockdown at 6, 12, and 18 hpi (Fig.

238 1B). To study the role of DDX21 in innate immunity, the expression levels of IFN- $\beta$

239 and downstream interferon-stimulate gene (ISG), interferon-induced protein with

240 tetratricopeptide repeats 1 (IFIT1), were evaluated. The results showed that the

241 knockdown of DDX21 inhibited the mRNA levels of IFN- $\beta$ (Fig. 1C) and IFIT-1 (Fig.

242 1D). Consistently, phosphorylation of TBK1 was significantly inhibited at 6,12 and

24318 hpi. Similar results were observed in DDX21 knockdown cells after treatment of

244 poly (I:C), confirmed the positive regulation of IFN- $\beta$ pathway by DDX21 (Fig.

245 1E-G). For further confirmation, we generated DDX21 knockout cells. Unfortunately,

246 after three rounds of screening, only one $(1 / 225)$ heterozygous clone $(d d x 21+/-)$ was

247 identified by sequencing (Fig. S1A). Western blotting showed that compared with

248 WT cells, DDX21 expression was significantly inhibited in ddx21+/- cells (Fig. S1B).

249 The regulation of virus titers and IFN- $\beta$ pathway was in accord with the knockdown

250 results (Fig. S1C-G). However, using the overexpression model, we observed that

251 transfection of exogenous DDX21 only increased VSV titers at 12 hpi (Fig. 1I), and 
252 the synthesis of virus $\mathrm{G}$ protein was unchanged at all time points pi (Fig. 1H).

253 Correspondingly, DDX21 overexpression had no effect on IFN- $\beta$ production or the

254 mRNA levels of ISGs (Fig. 1J-K). Collectively, these results suggested that DDX21

255 positively regulates IFN- $\beta$ signaling pathway. However, the discrepancy between the

256 knockdown and overexpression models raised the question of whether, in addition to

257 DDX21 expression, the modification of DDX21 plays a role in anti-viral innate

258 immunity.

The cleavage of DDX21 by virus infection and treatment with RNA/DNA ligands

260 Interestingly, in both the knockdown and overexpression experiments, we observed

261 the apparent cleavage of DDX21 in the course of VSV infection (Fig. 1A and H, Fig.

262 S1C). To further confirm the cleavage of DDX21 by virus infection, cells were

263 infected by two RNA viruses, VSV and NDV, and one DNA virus, HSV-1,

264 respectively, followed by DDX21 detection. As expected, VSV and NDV apparently

265 cleaved DDX21 at 12, 18, and 24 hpi. The full-length DDX21 almost disappeared at

26618 and 24 hpi post VSV and NDV infection (Fig. 2A and B). In comparison, HSV-1

267 only slightly cleaved DDX21, even at the late stage of infection, and the amount of

268 full-length DDX21 was not significantly decreased (Fig. 2C). Statistically, at 18 and

$26924 \mathrm{hpi}$, the ratio of DDX21/cleaved DDX21/ $\beta$-actin was 33-564 in VSV- and

270 NDV-infected cells, compared with 2.4-2.6 in HSV-1-infected cells (Fig. 2D-F).

271 A549, Huh7, and THP-1 cells were then utilized to test whether virus-triggered

272 DDX21 cleavage was cell type-dependent. The results showed that DDX21 was 
273 cleaved in A549, Huh7, or THP-1 cells upon VSV or NDV infection (Fig. S2A-D).

274 Additionally, the cleavage of DDX21 was observed in cells infected with SeV,

275 another RNA virus (Fig. S2E).

276 Given that DDX21 was cleaved upon RNA and DNA virus infection, and as

277 DDX21 belongs to the RNA helicase family that can bind various RNAs (22), we

278 propose that this cleavage is triggered by virus nucleotides. Two RNA ligands, poly

279 (I:C) and 3p-hpRNA, and two DNA ligands, poly (G:C) and HSV-60, were used to

280 evaluate their role in DDX21 cleavage. As expected, both RNA and DNA ligands

281 cleaved DDX21 (Fig. 2G and H), and higher cleavage efficiency was observed upon

282 treatment with RNA ligands, compared with that of DNA ligands (Fig. 2I and J),

283 which is in accordance with the results of virus infection. There are two transcript

284 variants of DDX21, isoform 1 (X1), which encodes the full-length of DDX21, and

285 isoform $2(\mathrm{X} 2)$, with a shorter $\mathrm{N}$-terminus ( $\Delta 1-86)$ compared to X1. Here, we showed

286 that both exogenously expressed DDX21 X1 and X2 were cleaved upon VSV, NDV,

287 and HSV-1 infection (Fig. 2H-J). Collectively, these results clearly demonstrated that

288 DDX21 was cleaved by virus infection and treatment with RNA/DNA ligands.

289 DDX21 was cleaved in a caspase-dependent manner

290 To further confirm whether DDX21 was cleaved or degraded upon virus infection and

291 RNA/DNA ligand treatment, cells were treated with caspase inhibitor z-VAD-FMK,

292 neddylation inhibitor MLN4924, proteasome inhibitor MG-132, and autophagy

293 inhibitors wortmannin and CQ, respectively, followed by virus or treatment with 
294 ligand. The results showed that DDX21 cleavage was completely inhibited after

295 treatment with the caspase inhibitor z-VAD-FMK in cells infected with VSV, NDV, 296 and HSV-1 (Fig. 3A-D, lane 4) or treated with RNA ligand poly (I:C) (Fig. 3D, lane 297 4). Notably, MG132 and CQ also seem to inhibit the cleavage of DDX21, especially 298 in VSV- and NDV-infected cells (Fig. 3A and B, lanes 6 and 7). However, the 299 expression of viral protein was also inhibited after treatment with these two drugs, 300 indicating that the inhibition of DDX21 cleavage may be due to their inhibition of 301 virus replication. In comparison, z-VAD-FMK had no effect on virus replication. 302 These results indicated that DDX21 was cleaved in a caspase-dependent manner.

\section{Caspase-3/6 cleaved DDX21 at $D^{126}$ in response to VSV infection}

304 DDX21 is characterized by several known domains, Q motif (amino acid (aa)

305 186-214), helicase ATP-binding domain (aa 217-396), and helicase C-terminal

306 domain (aa 429-573) (Fig. 4A). To characterize the cleavage sites of DDX21, based

307 on the identified domains, several deletion mutants $(\Delta 1-216, \Delta 217-395, \Delta 396-573$,

$308 \Delta 574-784)$ were generated to test the critical domain involved in DDX21 cleavage. As

309 shown in Fig. 4B, deletion of 1-216 abrogated the cleavage of DDX21. Notably,

310 unlike endogenous DDX21 expression, exogenous expression of DDX21 truncates

311 alone is able to induce cleavage to some extent (Fig. 4B, left panel). Similar results

312 could be observed in the overexpression of wild-type (WT) DDX21 (Fig. 2H-J).

313 Combined with the results showing that aa 1-216 is critical for DDX21 (Fig. 4B) and 314 aa 1-86 is not required for DDX21 cleavage (Fig. 2H-J), the cleavage sites are within 
315 aa 87-216 of DDX21. The caspase cleavage sites were then predicted by CaspDB

316 (http://caspdb.sanfordburnham.org). The results showed that all three aspartates (Asp,

317 D), D87, D126, D160, are the putative caspase cleavage sites (Fig. 4C). Therefore,

318 DDX21 with single, double, and triple mutations of these three Asp sites were

319 generated, respectively, followed by transfection and virus infection. The results

320 showed that D126A, but not D87A or D160A, is sufficient and necessary for DDX21

321 cleavage (Fig. 4D, lanes 4, 6, 8 and 9). The truncates of DDX21 1-125 and 127-784,

322 together with WT DDX21, were transfected into cells to further confirm that the

323 cleavage was mediated by D126. As expected, WT, but not 1-125 and 127-784

324 DDX21, was cleaved upon virus infection (Fig. 4E). It should be noted that based on

325 the molecular weight of DDX21, we proposed that the "cDDX21" we observed in

326 endogenous cleavage $(\sim 73 \mathrm{kD})$ was DDX21 127-784, while the relatively small

327 cleaved DDX21 ( 14kD) was degraded upon virus infection (Fig. 4E). Previous

328 reports showed that the cleavage sites specific for caspases had a general motif (37).

329 The motif for DDX21 cleavage is Glu-Ile-Asp (E-I-D), which is the putative substrate

330 for caspase-3 or caspase-6 (Fig. 4F). casp3-/- and casp6-/- cells were generated to

331 verify their role in DDX21 cleavage. As expected, knockout of casp3 and especially

332 casp6 significantly inhibited the cleavage of DDX21 (Fig. 4G-H). DDX21 cleavage

333 was almost blocked in casp3/6 double knockout cell lines (Fig. 4I). These results

334 indicated that virus infection cleaved DDX21 at D126 via caspase-3/6. 


\section{DDX21 was cleaved and translocated from the nucleus to the cytoplasm in}

338 DDX21 was identified to be a nucleolar helicase that is required for pre-rRNA

339 processing $(22,23,38)$. Other reports showed that DDX21 is localized with DDX1,

340 DDX26, and TRIF to sense dsRNA in the cytosol (20). To study whether DDX21

341 localization was affected by virus infection, cells were infected with VSV and HSV-1,

342 followed by IFA test. As expected, endogenous DDX21 was predominantly localized

343 in the nucleolus in mock-infected cells (Fig. 5A and C, upper panels). DDX21 was

344 translocated from the nucleolus to nucleoplasm and cytoplasm after VSV and HSV-1

345 infection (Fig. 5A and C, middle and lower panel). Statistically, at 12 and 24 hpi, cells

346 with cytoplasmic DDX21 accounted for $23 \%$ and $68 \%$ of all cells for VSV (Fig. 5B)

347 and $24 \%$ and $49 \%$ of all cells for HSV-1 (Fig. 5D), respectively, indicating that VSV

348 could induce DDX21 translocation more efficiently than HSV-1. Next, we evaluated

349 whether the cleavage of DDX21 affected its localization. WT, D126A, 1-125, and

350 127-784 DDX21 (Fig. 5E), together with empty vector, were transfected into cells

351 followed by VSV infection. The results showed that in mock-infected cells, WT,

352 D126A, and 127-784 DDX21 predominantly localized in the nucleolus (Fig. 5F, left

353 panel). WT and 127-784, but not D126A DDX21, efficiently translocated from the

354 nucleolus to nucleoplasm and cytoplasm after VSV infection (Fig. 5F and G),

355 indicating that blockage of DDX21 cleavage inhibited its translocation. Moreover, the

356 statistical analysis results showed the most efficient translocation of DDX21 cleaved 
form (cDDX21, 126-784), further indicating DDX21 cleavage promotes its

translocation from nucleus to cytoplasm. Interestingly, 1-125 DDX21 was diffusely

and G). Collectively, these results demonstrated that the virus induced the

361 translocation of DDX21, which is affected by DDX21 cleavage.

\section{DDX21 cleavage led to the inhibition of the IFN- $\beta$ signaling pathway}

363 Given that the blockage of DDX21 inhibits its translocation, we next aimed to explore

364 the effect of DDX21 cleavage in the regulation of innate immunity. $D d x 21+/-$ cells

365 were transfected with WT and truncated DDX21, followed by the detection of virus

366 replication and IFN- $\beta$ signaling pathway. Interestingly, transfection of WT and

367 truncated DDX21 did not affect virus replication, evidenced by viral protein

368 expression and titers in the supernatants (Fig. 6A and B). In contrast, although WT

369 DDX21 has no effect, the intact form of DDX21 (D126A) did increase the mRNA

370 levels of IFN- $\beta$ and ISGs (IFIT-1 and MX1) after VSV infection. More importantly,

371 transfection of cDDX21 significantly inhibited IFN- $\beta$ signaling pathway (Fig. 6C-E).

372 These results were further confirmed using poly (I:C) as the stimulator (Fig. 6F-H).

373 To identify whether DDX21 cleavage regulates IFN- $\beta$ signaling pathway in a

374 time-dependent manner, $d d x 21+/$ - cells stably expressing flag-tagged WT and D126A

375 DDX21 infected by VSV or treated with poly (I:C) and collected at different time

376 points. DDX21 was cleaved in WT but not D126A DDX21-expressed cells. However,

377 no difference was observed in viral protein synthesis between WT and D126A 
DDX21-expressed cells (Fig. 6I). More importantly, blockage the cleavage (D126A)

\section{Cleavage of DDX21 inhibited the formation of DDX1-DDX21-DHX36 complex}

Given that DDX21 was reported to act as the scaffold protein in the complex of three

hypothesized that DDX21 cleavage attenuates the inhibition of the IFN- $\beta$ signaling

pathway by inhibition of the formation of DDX1-DDX21-DHX36 complex. Since

DDX21 was reported to exist as a homodimer $(25,27)$, the cleavage of DDX21 on its self-interaction was studied first. As shown in Fig. 7A, using full-length DDX21 as

391 the bait, the full-length and 127-784, but not 1-125 DDX21, could be

392 immunoprecipitated (Fig. 7A). In accordance, 127-784 DDX21 was interacted with

393 127-784 and full-length, but not 1-125 DDX21 (Fig. 7B). In contrast, 1-125 DDX21

394 was not immunoprecipitated with WT and truncated DDX21 (Fig. 7C). These results

395 indicated that DDX21 interacted with itself through its C-terminal 127-784. Next, we

396 evaluated whether DDX21cleavage inhibits the formation of DDX1-DDX21-DHX36

397 complex. The results showed that compared with intact DDX21 (D126A), cDDX21

398 (127-784) has a lower affinity for both DDX1 and TRIF (Fig. 7E-F). In comparison, 
bioRxiv preprint doi: https://doi.org/10.1101/2021.04.06.438751; this version posted April 8, 2021. The copyright holder for this preprint (which was not certified by peer review) is the author/funder. All rights reserved. No reuse allowed without permission.

399 D126A, 127-784 and 1-125 DDX21 showed similar binding ability to DHX36 (Fig.

400 7G). Collectively, these results indicated cleavage of DDX21 inhibited the formation

401 of DDX1-DDX21-DHX36 complex and its interaction to downstream adaptor TRIF. 
402

403

\section{Discussion}

Intensive functional and structural research over the years has clearly

demonstrated that RLRs selectively bind viral RNA ligands and trigger downstream

signaling (40). Several other DExD/H-box helicases have been implicated in anti-viral innate immunity, but the fundamental questions such as the precise regulation mechanism how DExD/H-box helicases regulate innate immunity remain to be elucidated. Here, we demonstrated that DDX21 is cleaved at $\mathrm{D}^{126}$ after virus infection and RNA/DNA ligand treatment via the caspase-3/6 pathway, leading to the inhibition of immune responses.

To date, there has been limited research on the cleavage of RNA helicases.

Human RNA helicase A, a nuclear helicase that unwinds dsRNA, dsDNA, and RNA:DNA duplexes, was reported to be cleaved by caspase-3 during apoptosis (41). Another study also showed that the CARD-containing helicase (Helicard) is cleaved by caspases upon apoptotic stimuli (42). However, no studies have shown that the cleavage of DNA/RNA helicases is involved in innate immunity and virus infection.

Our data showed the obvious cleavage of DDX21 in response to virus infection and treatment with ligands. The fact that three RNA viruses and one DNA virus, together with two RNA and two DNA ligands, trigger varying degrees of DDX21 cleavage leads us to speculate that the ability of DDX21 cleavage may be shared by most viruses. 
423 immune signaling (43). In terms of the innate immunity pathway, caspase- 1 is one of

424 the most well-studied caspases, which is able to cleave proIL-1b and proIL-18 and

425 triggers inflammasomes (44). It has also been reported that caspases are involved in

426 the RLR-mediated type I IFN response. For instance, caspase-12 positively modulates

427 the IFN- $\beta$ signaling pathway by regulating E3 ubiquitin ligase TRIM25-mediated

428 ubiquitination of RIG-I (45). Activated caspase-3/7/9 suppress mitochondrial

429 DNA-induced stimulator of interferon genes protein (STING)-mediated type I IFN

430 production (46). Here, we showed that the cleavage of DDX21 was completely

431 recovered in the presence of caspase inhibitor z-VAD-FMK (Fig. 3), and the cleavage

432 was probably mediated by caspase-3/6 (Fig. 4). It should be noted that knockout of

433 casp3 and casp6 did not completely blocked DDX21 cleavage, indicating that the

434 involvement of other caspases could not be completely excluded. A recent study

435 revealed that caspase-6 cleaves IL-1R-associated kinase-M (IRAK-M) and reduces

436 I $\kappa \mathrm{B} \alpha$ degradation, thereby increasing TNF- $\alpha$ production (47). The function of

437 caspase-6 in the type I IFN pathway has not been reported. Our study indicated that

438 caspase-3/6 cleaves DDX21 and thus likely regulates the IFN- $\beta$ signaling pathway.

439 In a resting state, DDX21, together with its binding partners, c-Jun, WDR46,

440 SIRT7, etc., is localized in the cell nucleolus $(23,38,48)$. The nucleolar localization

441 of DDX21 is necessary for its pre-rRNA processing and RNA unwinding $(23,48)$.

442 Studies have also suggested that DDX21 is involved in innate immunity in the 

to the mitochondria upon poly(I:C) stimulation (20). The infection of A549 cells with the dengue virus causes DDX21 to partially relocate from the nucleus to the cytoplasm (29). Here, we confirmed that DDX21 localized in the cell nucleolus in mock-infected cells. Virus infection effectively triggered DDX21 translocation from

448 the nucleolus to the cytoplasm. In resting cells, WT, D126A, and 127-784 DDX21

449 showed nucleolar localization. It is interesting that 1-125 DDX21 diffusely localized

450 in the nucleus and the cytoplasm. Previous reports showed that the 731-740 at the

451 C-terminus of DDX21 interacted with c-Jun, and depletion of c-Jun promotes DDX21

452 translocation from the nucleolus to the nucleoplasm (23). Therefore, it is possible that

453 the deletion of the C-terminus of DDX21 abolishes the interaction with its binding

454 partner and thus alters its nucleolar localization. Our results that (1) blockage of

455 DDX21 cleavage inhibits DDX21 translocation, (2) the most efficient translocation of

456 cDDX21 upon virus infection, indicated that cleavage of DDX21 promotes its

457 translocation from nucleus to cytoplasm.

Various DDX and DHX helicases play important roles in maintaining the stability of the cell genome $(22,48,49)$. To date, the knockout of $d d x 21$ has never

460 been reported in cells or mice. Studies on other DDXs have also shown that certain

461 DDXs are critical for mouse growth, and knockout of the $d d x$ gene results in early

462 embryonic lethality $(50,51)$. Therefore, DDX21 may also be critical for cell and

463 mouse survival. Nevertheless, DDX21 expression was significantly inhibited in 
influence virus replication in other ways except for innate immunity. Nevertheless, it

infection, which lead us to speculate the cleavage of DDX21 was driven by host for a

\section{late counter-regulatory effect to temper immune responses.}

477 between these key pathways is essential for immune homeostasis. Indeed, DNA

478 sensor cGAS has been reported as a key driver of lethal autoimmune disease in the

479 Trex1-deficient mouse model of Aicardi-Goutieres Syndrome (AGS) (55). The

480 importance of excess RLR-dependent signaling, which leads to an IFN signature in

481 the pathogenesis of many autoimmune diseases, such as AGS and systemic lupus

482 erythematosus, has also been clarified (56). These reports highlight the importance of

483 fine-tuning the regulation of the type I IFN signaling pathway. Here, we provided 
491 from our original and additional data, we inferred that host promotes DDX21

cleavage via caspase3/6 to suppress DDX1-DDX21-DHX36 complex formation for a activities. 


\section{Acknowledgements}

500 IFN- $\beta$-Luc. We thank Dr. Yasushi Kawaguchi (university of Tokyo, Japan) for

501 providing HSV-1. We thank Dr. Jianchao Wei (Shanghai Veterinary Research

502 Institute, China) for providing VSV. We thank Quan Zhang (Yangzhou University,

503 China) for providing Sendai virus (SeV). 


\section{Funding information}

505 This work was funded by grants 32030108 (to C. D) and 31872453 (to Y. S) from the

506 National Natural Science Foundation of China and by grant 2018 YFD0500100 from

507 the National Key Research and Development Program of China to C. D. The funders

508 had no role in study design, data collection and interpretation, or the decision to

509 submit the work for publication.

510 


\section{Reference}

512 1. Takeuchi O, Akira S. 2010. Pattern recognition receptors and inflammation. Cell 140:805-20.

2. Alexopoulou L, Holt AC, Medzhitov R, Flavell RA. 2001. Recognition of double-stranded RNA and activation of NF-kappaB by Toll-like receptor 3 . Nature 413:732-8.

3. Yamamoto M, Sato S, Mori K, Hoshino K, Takeuchi O, Takeda K, Akira S. 2002. Cutting edge: a novel Toll/IL-1 receptor domain-containing adapter that preferentially activates the IFN-beta promoter in the Toll-like receptor signaling. J Immunol 169:6668-72.

6. Xu LG, Wang YY, Han KJ, Li LY, Zhai Z, Shu HB. 2005. VISA is an adapter protein required for virus-triggered IFN-beta signaling. Mol Cell 19:727-40. Reis e Sousa C, Matsuura Y, Fujita T, Akira S. 2006. Differential roles of MDA5 and RIG-I helicases in the recognition of RNA viruses. Nature

5. Yoneyama M, Kikuchi M, Natsukawa T, Shinobu N, Imaizumi T, Miyagishi M, Taira K, Akira S, Fujita T. 2004. The RNA helicase RIG-I has an essential function in double-stranded RNA-induced innate antiviral responses. Nat Immunol 5:730-7. specific and essential role of MAVS in antiviral innate immune responses.

544 11. Patel SS, Donmez I. 2006. Mechanisms of helicases. J Biol Chem

\section{$545 \quad 281: 18265-8$.}

546 12. Umate P, Tuteja N, Tuteja R. 2011. Genome-wide comprehensive analysis of 547 human helicases. Commun Integr Biol 4:118-37.

548 13. Singleton MR, Dillingham MS, Wigley DB. 2007. Structure and mechanism 549 of helicases and nucleic acid translocases. Annu Rev Biochem 76:23-50.

550 14. Steimer L, Klostermeier D. 2012. RNA helicases in infection and disease. $551 \quad$ RNA Biol 9:751-71. 
552

553

554

555

556

557

558

559

560

561

562

563

564

565

566

567

568

569

570

571

572

573

574

575

576

577

578

579

580

581

582

583

584

585

586

587

588

589

590

591

15. Fairman-Williams ME, Guenther UP, Jankowsky E. 2010. SF1 and SF2

helicases: family matters. Curr Opin Struct Biol 20:313-24.

16. Ariumi Y, Kuroki M, Abe K, Dansako H, Ikeda M, Wakita T, Kato N. 2007. DDX3 DEAD-box RNA helicase is required for hepatitis $\mathrm{C}$ virus RNA replication. J Virol 81:13922-6.

17. Miyashita M, Oshiumi H, Matsumoto M, Seya T. 2011. DDX60, a DEXD/H box helicase, is a novel antiviral factor promoting RIG-I-like receptor-mediated signaling. Mol Cell Biol 31:3802-19.

18. Oshiumi H, Ikeda M, Matsumoto M, Watanabe A, Takeuchi O, Akira S, Kato N, Shimotohno K, Seya T. 2010. Hepatitis C virus core protein abrogates the DDX3 function that enhances IPS-1-mediated IFN-beta induction. PLoS One 5:e14258.

19. Randall G, Panis M, Cooper JD, Tellinghuisen TL, Sukhodolets KE, Pfeffer S, Landthaler M, Landgraf P, Kan S, Lindenbach BD, Chien M, Weir DB, Russo JJ, Ju J, Brownstein MJ, Sheridan R, Sander C, Zavolan M, Tuschl T, Rice CM. 2007. Cellular cofactors affecting hepatitis $\mathrm{C}$ virus infection and replication. Proc Natl Acad Sci U S A 104:12884-9.

20. Zhang Z, Kim T, Bao M, Facchinetti V, Jung SY, Ghaffari AA, Qin J, Cheng G, Liu YJ. 2011. DDX1, DDX21, and DHX36 helicases form a complex with the adaptor molecule TRIF to sense dsRNA in dendritic cells. Immunity 34:866-78.

21. Zhang Z, Yuan B, Bao M, Lu N, Kim T, Liu YJ. 2011. The helicase DDX41 senses intracellular DNA mediated by the adaptor STING in dendritic cells. Nat Immunol 12:959-65.

22. Calo E, Flynn RA, Martin L, Spitale RC, Chang HY, Wysocka J. 2015. RNA helicase DDX21 coordinates transcription and ribosomal RNA processing. Nature 518:249-53.

23. Holmstrom TH, Mialon A, Kallio M, Nymalm Y, Mannermaa L, Holm T, Johansson H, Black E, Gillespie D, Salminen TA, Langel U, Valdez BC, Westermarck J. 2008. c-Jun supports ribosomal RNA processing and nucleolar localization of RNA helicase DDX21. J Biol Chem 283:7046-53.

24. Henning D, So RB, Jin R, Lau LF, Valdez BC. 2003. Silencing of RNA helicase II/Gualpha inhibits mammalian ribosomal RNA production. J Biol Chem 278:52307-14.

25. Flores-Rozas H, Hurwitz J. 1993. Characterization of a new RNA helicase from nuclear extracts of HeLa cells which translocates in the $5^{\prime}$ to 3 ' direction. J Biol Chem 268:21372-83.

26. McRae EKS, Davidson DE, Dupas SJ, McKenna SA. 2018. Insights into the RNA quadruplex binding specificity of DDX21. Biochim Biophys Acta Gen Subj 1862:1973-1979. 
27. McRae EKS, Booy EP, Moya-Torres A, Ezzati P, Stetefeld J, McKenna SA. 2017. Human DDX21 binds and unwinds RNA guanine quadruplexes. Nucleic Acids Res 45:6656-6668.

28. Valdez BC, Henning D, Perumal K, Busch H. 1997. RNA-unwinding and RNA-folding activities of RNA helicase II/Gu--two activities in separate domains of the same protein. Eur J Biochem 250:800-7.

29. Dong Y, Ye W, Yang J, Han P, Wang Y, Ye C, Weng D, Zhang F, Xu Z, Lei Y. 2016. DDX21 translocates from nucleus to cytoplasm and stimulates the innate immune response due to dengue virus infection. Biochem Biophys Res Commun 473:648-53.

30. Chen G, Liu CH, Zhou L, Krug RM. 2014. Cellular DDX21 RNA helicase inhibits influenza A virus replication but is counteracted by the viral NS1 protein. Cell Host Microbe 15:484-93.

31. Sun Y, Yu S, Ding N, Meng C, Meng S, Zhang S, Zhan Y, Qiu X, Tan L, Chen H, Song C, Ding C. 2014. Autophagy benefits the replication of Newcastle disease virus in chicken cells and tissues. J Virol 88:525-37.

32. Li X, Qiu Y, Shen Y, Ding C, Liu P, Zhou J, Ma Z. 2008. Splicing together different regions of a gene by modified polymerase chain reaction-based site-directed mutagenesis. Anal Biochem 373:398-400.

33. Zheng L, Baumann U, Reymond JL. 2004. An efficient one-step site-directed and site-saturation mutagenesis protocol. Nucleic Acids Res 32:e115.

34. Xu W, Zhang Z, Zou K, Cheng Y, Yang M, Chen H, Wang H, Zhao J, Chen P, He L, Chen X, Geng L, Gong S. 2017. MiR-1 suppresses tumor cell proliferation in colorectal cancer by inhibition of Smad3-mediated tumor glycolysis. Cell Death Dis 8:e2761.

35. Sun Y, Ding N, Ding SS, Yu S, Meng C, Chen H, Qiu X, Zhang S, Yu Y, Zhan Y, Ding C. 2013. Goose RIG-I functions in innate immunity against Newcastle disease virus infections. Mol Immunol 53:321-7.

36. Schmittgen TD, Livak KJ. 2008. Analyzing real-time PCR data by the comparative $\mathrm{C}(\mathrm{T})$ method. Nat Protoc 3:1101-8.

37. McStay GP, Salvesen GS, Green DR. 2008. Overlapping cleavage motif selectivity of caspases: implications for analysis of apoptotic pathways. Cell Death Differ 15:322-31.

38. Hirai Y, Louvet E, Oda T, Kumeta M, Watanabe Y, Horigome T, Takeyasu K. 2013. Nucleolar scaffold protein, WDR46, determines the granular compartmental localization of nucleolin and DDX21. Genes Cells 18:780-97.

39. Gantier MP, Williams BR. 2011. Making sense of viral RNA sensing. Mol Ther 19:1578-81.

40. Yoneyama M, Onomoto K, Jogi M, Akaboshi T, Fujita T. 2015. Viral RNA detection by RIG-I-like receptors. Curr Opin Immunol 32:48-53. 
41. Takeda Y, Caudell P, Grady G, Wang G, Suwa A, Sharp GC, Dynan WS, Hardin JA. 1999. Human RNA helicase A is a lupus autoantigen that is cleaved during apoptosis. J Immunol 163:6269-74.

42. Kovacsovics M, Martinon F, Micheau O, Bodmer JL, Hofmann K, Tschopp J. 2002. Overexpression of Helicard, a CARD-containing helicase cleaved during apoptosis, accelerates DNA degradation. Curr Biol 12:838-43.

43. Creagh EM. 2014. Caspase crosstalk: integration of apoptotic and innate immune signalling pathways. Trends Immunol 35:631-640.

44. Latz E, Xiao TS, Stutz A. 2013. Activation and regulation of the inflammasomes. Nat Rev Immunol 13:397-411.

45. Wang P, Arjona A, Zhang Y, Sultana H, Dai J, Yang L, LeBlanc PM, Doiron K, Saleh M, Fikrig E. 2010. Caspase-12 controls West Nile virus infection via the viral RNA receptor RIG-I. Nat Immunol 11:912-9.

46. White MJ, McArthur K, Metcalf D, Lane RM, Cambier JC, Herold MJ, van Delft MF, Bedoui S, Lessene G, Ritchie ME, Huang DC, Kile BT. 2014. Apoptotic caspases suppress mtDNA-induced STING-mediated type I IFN production. Cell 159:1549-62.

47. Kobayashi H, Nolan A, Naveed B, Hoshino Y, Segal LN, Fujita Y, Rom WN, Weiden MD. 2011. Neutrophils activate alveolar macrophages by producing caspase-6-mediated cleavage of IL-1 receptor-associated kinase-M. J Immunol 186:403-10.

48. Song C, Hotz-Wagenblatt A, Voit R, Grummt I. 2017. SIRT7 and the DEAD-box helicase DDX21 cooperate to resolve genomic R loops and safeguard genome stability. Genes Dev 31:1370-1381.

49. Hodroj D, Serhal K, Maiorano D. 2017. Ddx19 links mRNA nuclear export with progression of transcription and replication and suppresses genomic instability upon DNA damage in proliferating cells. Nucleus 8:489-495.

50. Ma Z, Moore R, Xu X, Barber GN. 2013. DDX24 negatively regulates cytosolic RNA-mediated innate immune signaling. PLoS Pathog 9:e1003721.

51. Janknecht R. 2010. Multi-talented DEAD-box proteins and potential tumor promoters: p68 RNA helicase (DDX5) and its paralog, p72 RNA helicase (DDX17). Am J Transl Res 2:223-34.

52. Xing YH, Yao RW, Zhang Y, Guo CJ, Jiang S, Xu G, Dong R, Yang L, Chen LL. 2017. SLERT Regulates DDX21 Rings Associated with Pol I Transcription. Cell 169:664-678 e16.

53. Nallar SC, Kalvakolanu DV. 2014. Interferons, signal transduction pathways, and the central nervous system. J Interferon Cytokine Res 34:559-76.

54. Crow MK. 2014. Advances in understanding the role of type I interferons in systemic lupus erythematosus. Curr Opin Rheumatol 26:467-74.

55. Gray EE, Treuting PM, Woodward JJ, Stetson DB. 2015. Cutting Edge: cGAS Is Required for Lethal Autoimmune Disease in the Trex1-Deficient Mouse Model of Aicardi-Goutieres Syndrome. J Immunol 195:1939-43. 
bioRxiv preprint doi: https://doi.org/10.1101/2021.04.06.438751; this version posted April 8, 2021. The copyright holder for this preprint (which was not certified by peer review) is the author/funder. All rights reserved. No reuse allowed without permission.

674 56. Kato H, Fujita T. 2015. RIG-I-like receptors and autoimmune diseases. Curr $675 \quad$ Opin Immunol 37:40-5.

676 
679 (A) HeLa cells with stable knockdown of DDX21 and control cells were mock treated or infected with VSV at an MOI of 1, respectively. Cells were harvested at anti-VSV-G, anti-p-TBK1, anti-TBK1 or anti- $\beta$-actin antibody.

(B) Extracellular virus yields in DDX21 knockdown and control cells. and detected using qRT-PCR with IFN- $\beta$ (C) and IFIT-1(D) primers, respectively. anti-DDX21, anti-VSV-G, anti-p-TBK1, anti-TBK1 or anti- $\beta$-actin antibody. respectively. after transfection, cells were mock treated or infected with VSV at an MOI of 1. Cells were harvested at $6,12,18$, and $24 \mathrm{hpi}$, and detected using immunoblot analysis with anti-DDX21, anti-VSV-G, or anti- $\beta$-actin antibody. 

and detected using qRT-PCR with IFN- $\beta(\mathrm{J})$ and IFIT-1(K) primers, respectively.

700 Data are presented as means from three independent experiments. *, $\mathrm{P}<0.05$, **, $\mathrm{P}<0.01, * * *, \mathrm{P}<0.001$ control condition for VSV (D), NDV (E) and HSV-1 (F) infection group. Data are

711 presented as means from three independent experiments. ***, $\mathrm{P}<0.001$.

712 (G-H) HeLa cells were transfected with RNA ligands (poly (I:C) or 3p-hpRNA, G)

713 or DNA ligands (poly (G:C) or HSV-60, H), respectively. At 18 hours post

714 tranfection (hpt), cells were harvested and detected using immunoblot analysis

715 with anti-DDX21 or anti- $\beta$-actin antibody.

716 (I-J) Representative results are shown with graphs representing the intensity band 717 ratio of cleaved DDX21 (cDDX21)/DDX21/ $\beta$-actin normalized to the control 718 condition for RNA (I) and DNA (J) ligand treatments group. 
719 (K) Schematic representation of two transcript isoforms of DDX21.

720 (H-J) HeLa cells were transfected with either empty vector, Flag-DDX21-X1,

721 Flag-DDX21-X2. At $24 \mathrm{~h}$ after transfection, cells were mock treated or infected

722 with VSV (H), NDV (I) or HSV-1 (J), respectively, at an MOI of 1. Cells were

723 harvested at $18 \mathrm{hpi}$, and detected using immunoblot analysis with anti-DDX21,

724 anti- $\beta$-actin or anti-viral-protein (VSV-G, NDV-NP, HSV-1-gD) antibody.

725 Figure 3. The caspase-dependent cleavage of DDX21 triggered by virus infection

726 or treatmens of RNA ligands

727 (A-C) HeLa cells were mock infected or infected with VSV(A), NDV (B) or

HSV-1 (C) at an MOI of 1 and maintained in the presence of DMSO control,

z-VAD-FMK (VAD), MLN4924 (MLN), MG132 (MG), CQ or wortmannin

(Wort) for $18 \mathrm{~h}$. Cells were harvested and detected using immunoblot analysis with with anti-DDX21, anti- $\beta$-actin or anti-viral-protein (VSV-G, HSV-1-gD, NDV-NP) antibody.

(D) HeLa cells were mock treated or transfected with RNA ligands (poly (I:C) and maintained in the presence of DMSO control, z-VAD-FMK, MLN4924, MG132, CQ or wortmannin for $18 \mathrm{~h}$. Cells were harvested and detected using immunoblot analysis with with anti-DDX21 or anti- $\beta$-actin antibody.

737 Figure 4. DDX21 was cleaved at $D^{126}$ by caspase-3/6 in response to VSV infection

738 (A) Schematic representation of WT DDX21 and its deletion mutants. 
739 (B) HeLa cells were transfected with Flag tagged DDX21 truncates $(\Delta 1-216$,

$740 \quad \Delta 217-395, \Delta 396-573, \Delta 574-784)$. At $24 \mathrm{~h}$ after transfection, cells were mock

741 treated or infected with VSV at an MOI of 1. Cells were harvested at $18 \mathrm{hpi}$, and

742 detected using immunoblot analysis with anti-Flag, anti-VSV-G, or anti- $\beta$-actin

743 antibody.

744 (C) The prediction results of caspase cleavage sites for DDX21 based on CaspDB

745 (http://caspdb.sanfordburnham.org). The red frame indicates aa 87-216 of DDX21.

746 The red asterisks $(*)$ indicate the putative caspase cleavage sites.

747 (D) HeLa cells were transfected with either empty vector or Flag tagged WT and

748 mutants of DDX21 (D87A, D126A, 160A, D87A/D126A, D87A/160A,

749 D126A/D160A, D81A/D126A/160A). At $24 \mathrm{~h}$ after transfection, cells were mock

750 treated or infected with VSV at an MOI of 1. Cells were harvested at 18 hpi, and

751 detected using immunoblot analysis with anti-Flag, anti-VSV-G, or anti- $\beta$-actin

752 antibody.

753 (E) HeLa cells were transfected with either empty vector or Flag tagged WT and

754 truncates of DDX21 (1-125, 127-784). At $24 \mathrm{~h}$ after transfection, cells were mock

755 treated or infected with VSV at an MOI of 1. Cells were harvested at $18 \mathrm{hpi}$, and

756 detected using immunoblot analysis with anti-DDX21, anti-VSV-G, or

$757 \quad$ anti- $\beta$-actin antibody.

758 (F) Schematic representation of amino acids around cleavage site. The red frame

759 indicates the general motif for caspase cleavage. 
761 HeLa cells were mock treated or infected with VSV at an MOI of 1, respectively.

762 Cells were harvested at $18 \mathrm{hpi}$, and detected using immunoblot analysis with

763 anti-DDX21, anti-caspase-3/6, anti-VSV-G, or anti- $\beta$-actin antibody.

764 Representative results are shown with graphs representing the intensity band ratio

765 of cleaved DDX21 (cDDX21)/DDX21/ $\beta$-actin normalized to the control

766 condition.

767 Figure 5. Virus infection triggers the translocation of DDX21 from nucleus to

768 cytoplasm which is dependent on its cleavage

769 (A, C) HeLa cells were mock treated or infected with VSV (A) or HSV-1 (C) at

$770 \quad$ an MOI of 1 . At 6 and 12 hpi, cells were fixed, and processed for IF using

771 anti-DDX21 or anti-viral-protein (VSV-G, HSV-1-gD) antibody. Nuclei were

$772 \quad$ stained with $1 \mu \mathrm{g} / \mathrm{mL}$ of DAPI.

773 (B, D) The relative percentages of cells with nuclear and cytoplasmic DDX21

774 staining upon VSV (B) or HSV-1 (D) infection were quantified. 10 images in 20

775 high-powered fields (HPFs) were obtained randomly in different fields.

776 (F) HeLa cells were transfected with either empty vector or Flag tagged WT,

777 D126A, 1-126 or 127-784 DDX21. At 24 h after transfection, cells were mock

778 treated or infected with VSV at an MOI of 1. At $12 \mathrm{hpi}$, cells were fixed, and

779 processed for IF using anti-Flag or anti-VSV-G antibody. Nuclei were stained

$780 \quad$ with $1 \mu \mathrm{g} / \mathrm{mL}$ of DAPI. 
(G) The relative percentages of cells with nuclear and cytoplasmic DDX21

(A) $d d x 21+/-$ HeLa cells were transfected with either empty vector or Flag tagged respectively. tagged WT, D126A, 1-126 or 127-784 DDX21. At $24 \mathrm{~h}$ after transfection, cells were mock treated or transfected with poly (I:C) $(20 \mu \mathrm{g} / \mathrm{mL})$, respectively. Cells were harvested at $12 \mathrm{hpt}$ and detected using qRT-PCR with IFN- $\beta$ (F), IFIT-1 (G), or MX1 (H) primers. 
801 (I) $d d x 21+/$ - cells stably expressing flag-tagged WT and D126A DDX21 were

802 mock treated or infected with VSV at an MOI of 1, respectively. Cells were

803 harvested at 6,12, 18 and $24 \mathrm{hpi}$, and detected using immunoblot analysis with

804 anti-DDX21, anti-VSV-G, or anti- $\beta$-actin antibody.

805 (J) Extracellular virus yields in $d d x 21+/$ - cells stably expressing flag-tagged WT

806 and D126A DDX21

$807 \quad$ (K) $d d x 21+/$ - cells stably expressing flag-tagged WT and D126A DDX21 were

808 co-transfected with IFN- $\beta$-Luc and PRL-TK. At $12 \mathrm{hpt}$, cells were mock treated or

809 infected with VSV at an MOI of 1, respectively. Cells were harvested at 6, 12 and

$81018 \mathrm{hpi}$ and assessed for luciferase activity. The results are presented as relative

$811 \quad$ luciferase activity.

812 (L-N) $d d x 21+/$ - cells stably expressing flag-tagged WT and D126A DDX21 were

813 mock treated or infected with VSV at an MOI of 1, respectively. Cells were

814 harvested at 6, 12 and 18 hpi and detected using qRT-PCR with IFN- $\beta$ (L), IFIT-1

$815(\mathrm{M})$, or MX1 (N) primers. The results are presented as relative luciferase activity.

816 (O) $d d x 21+/$ - cells stably expressing flag-tagged WT and D126A DDX21 were

817 co-transfected with p-125Luc and PRL-TK. At $12 \mathrm{hpt}$, cells were mock treated or

818 transfected with poly (I:C) $(20 \mu \mathrm{g} / \mathrm{mL})$. Cells were harvested at 6,12 and $18 \mathrm{hpt}$

819 and assessed for luciferase activity. The results are presented as relative luciferase

820 activity. 


$$
\mathrm{P}<0.01, * * *, \mathrm{P}<0.001
$$

Figure 7. DDX21 cleavage inhibits its interaction with DDX1 and TRIF asterisk $(*)$ indicates the predicted size of HA-DDX21 (1-125). 

anti-Flag antibody.

846 Figure 8. Proposed model for the regulation of innate immunity by DDX21 cleavage

847 during virus infection

The double-edged sword role of DDX21 in regulation of innate immunity upon

and recruit DDX21 and DHX36 to form a complex to mediate downstream anti-viral

innate immunity. At the late stage of infection, to avoid excessive immune response,

host promotes DDX21 cleavage via caspase3/6. Cleaved DDX21 tends to translocate 


\section{Supporting Information Legends}

S1 Table. Primers and siRNAs used in this study

859 (A) Confirmation of the genome editing by sequencing of the PCR amplicon from the

860 DDX21 genome of the cell lines.

861 (B) WT and $d d x 21+/-$ HeLa cells were seeded in 6-well plates and collected for immunoblot analysis with anti-DDX21, or anti- $\beta$-actin antibody.

(C) WT and $d d x 21+/-$ HeLa cells were mock treated or infected with VSV at an MOI

(D) Extracellular virus yields in DDX21 knockdown and control cells. respectively.

S2 Figure. The cleavage of DDX21 by virus infection in various cell types

871 (A-B) A549 cells were mock treated or infected with VSV (A) or NDV (B),

872 respectively, at an MOI of 1 . Cells were harvested at 6, 12, 18, and 24 hpi, and

873 detected using immunoblot analysis with anti-DDX21, anti- $\beta$-actin or

874 anti-viral-protein (VSV-G, NDV-NP) antibody.

875 (C-D) Huh7 (C) or THP-1 (D) cells were infected with VSV. The virus infection

876 experiments were performed as in A. 
877 (E) HeLa cells were infected with Sendai virus (SeV). The virus infection

878 experiments were performed as in A.

879 S3 Figure. Subcellular distribution of WT and truncated DDX21 by

880 nucleocytoplasmic separation assay

$881 d d \times 21+/-$ HeLa cells were transfected with either empty vector or Flag tagged WT,

882 D126A, 1-126 or 127-784 DDX21. At $24 \mathrm{~h}$ after transfection, cells were mock treated

883 or infected with VSV at an MOI of 1 . At $18 \mathrm{hpi}$, cells were harvested for

884 nucleocytoplasmic separation assay, and detected using immunoblot analysis with anti-Flag, anti- $\beta$-actin or anti-LaminB1 antibody.

886 
A

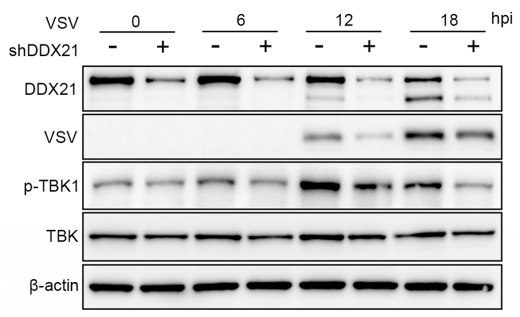

E

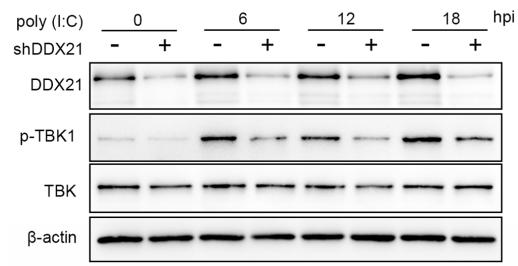

H

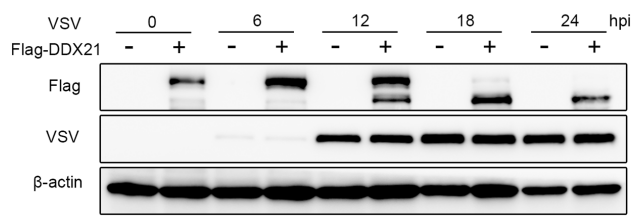

B
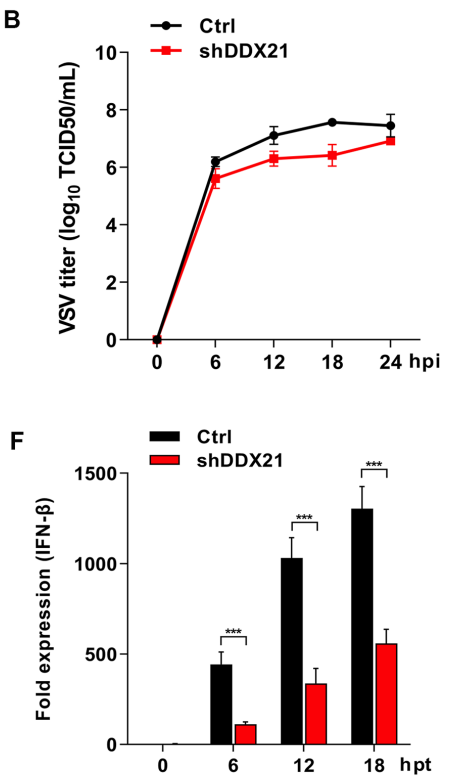

I

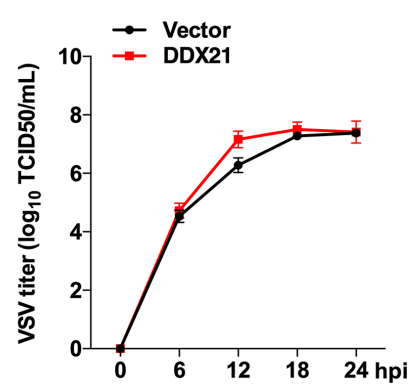

c

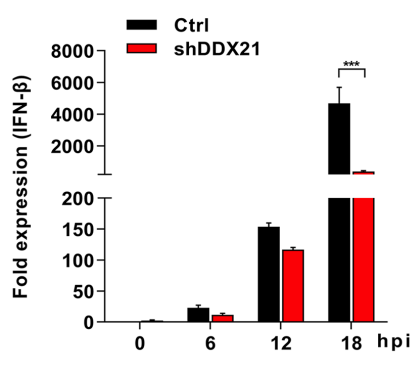

G
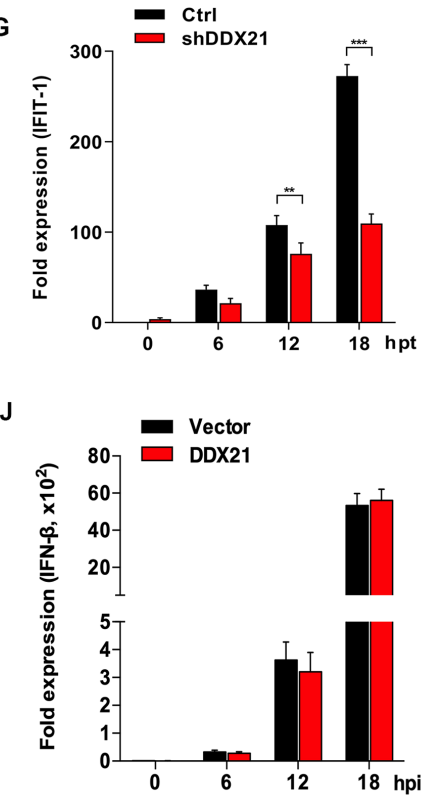
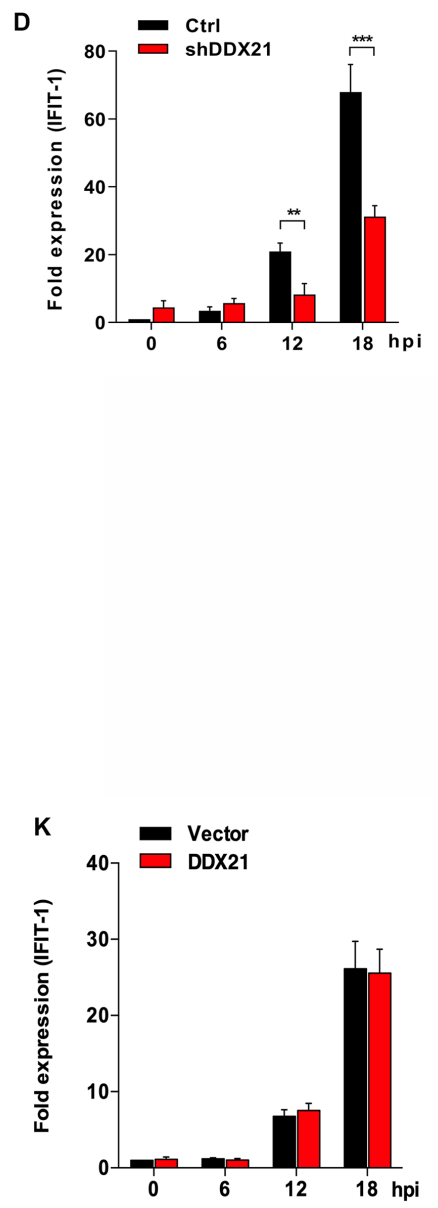
A

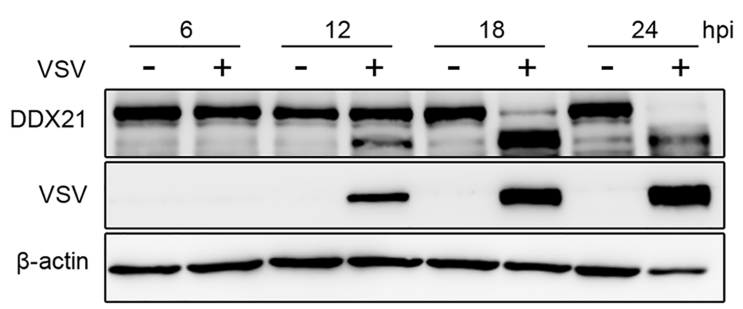

C

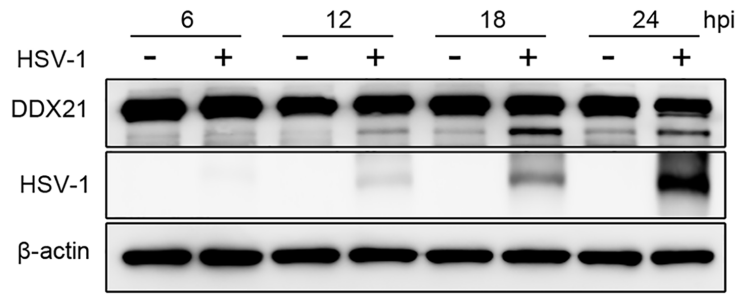

B

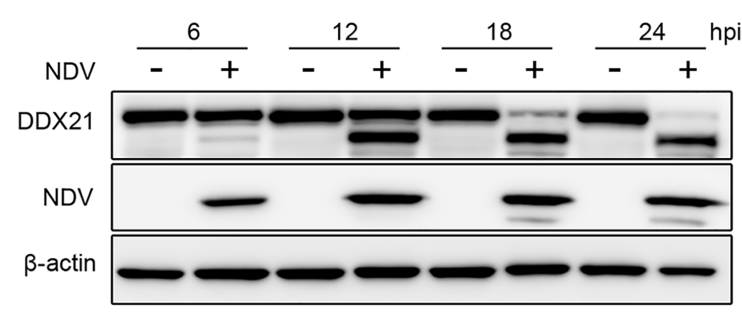

D

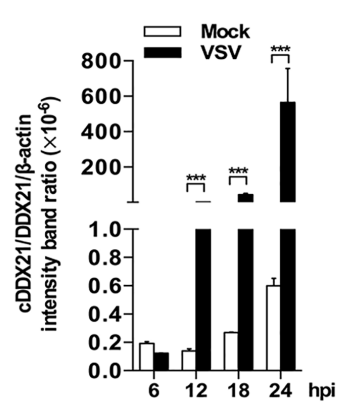

E

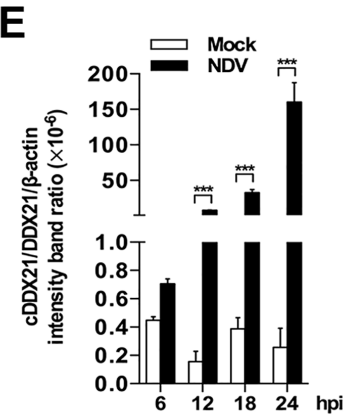

F

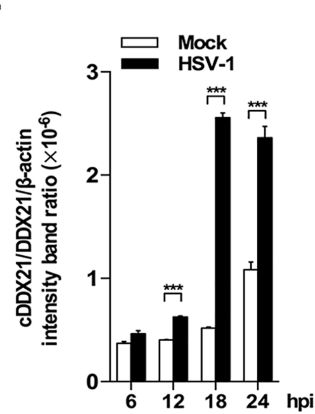

G

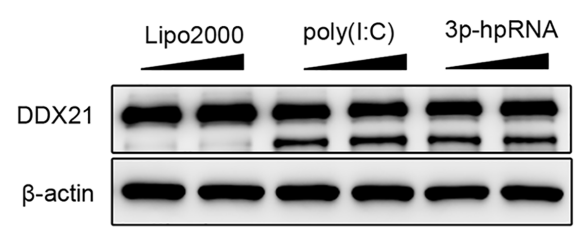

H

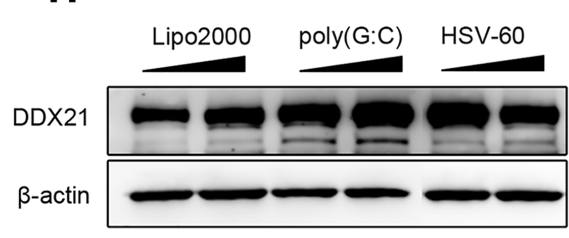

K

\begin{tabular}{|c|c|c|c|c|c|}
\hline \multirow{3}{*}{ DDX21 X1 } & & & & $396 \quad 4$ & 29 \\
\hline & 1 & Q & Helicase ATP-binding & & Helicase C-terminal \\
\hline & & 21 & & 3964 & 29 \\
\hline DDX21 X2 & & $\begin{array}{l}\text { Q } \\
\text { otif }\end{array}$ & Helicase ATP-binding & & Helicase C-terminal \\
\hline
\end{tabular}
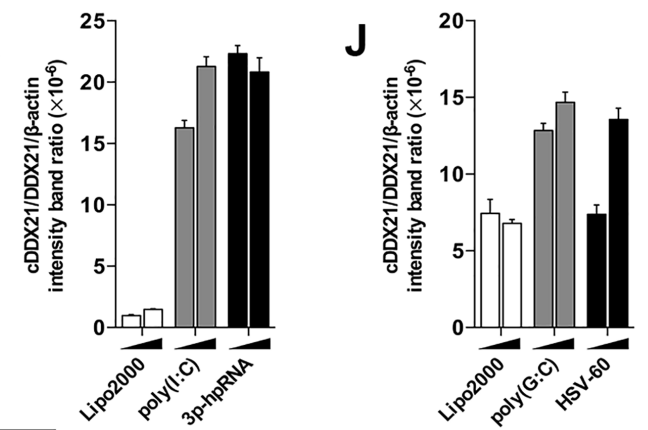
748

H

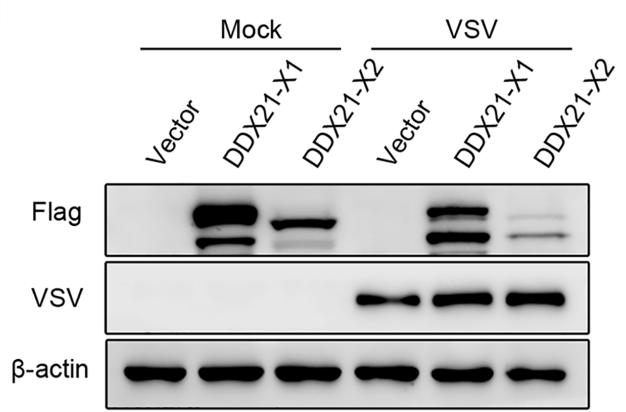

I
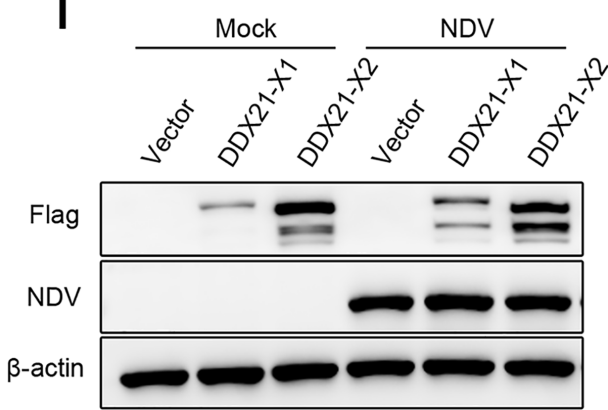

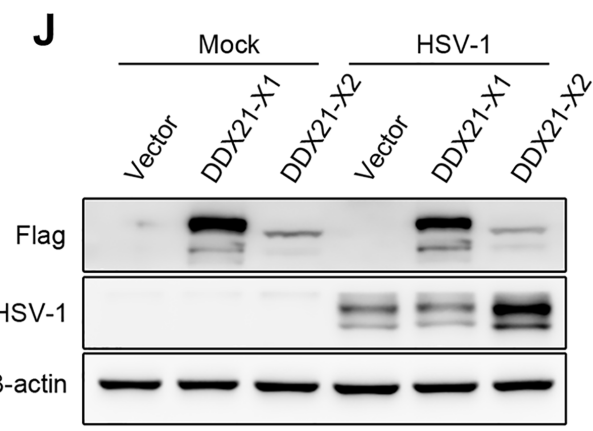


A

VSV

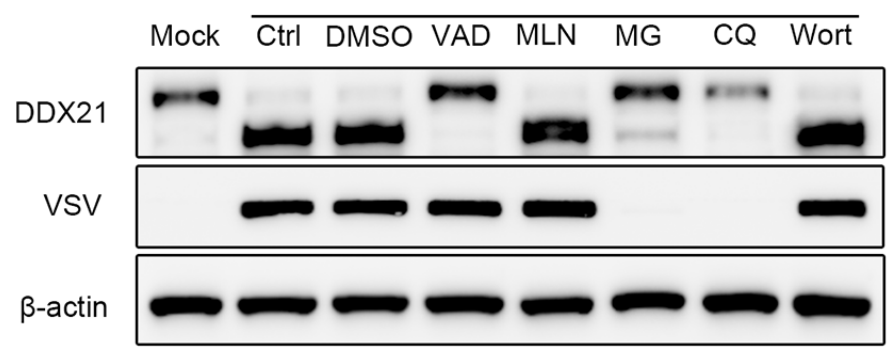

C

HSV-1

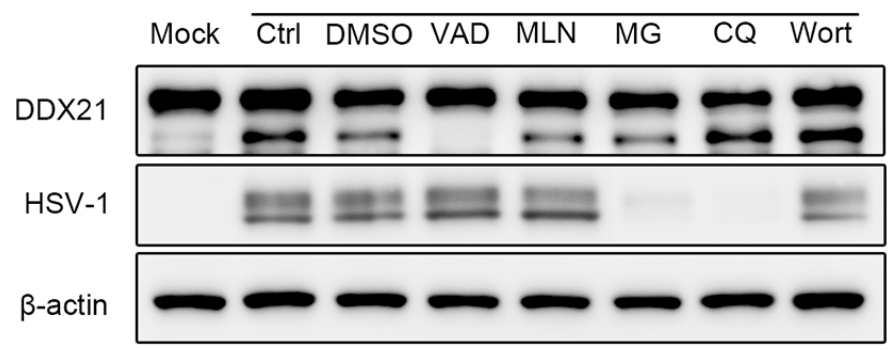

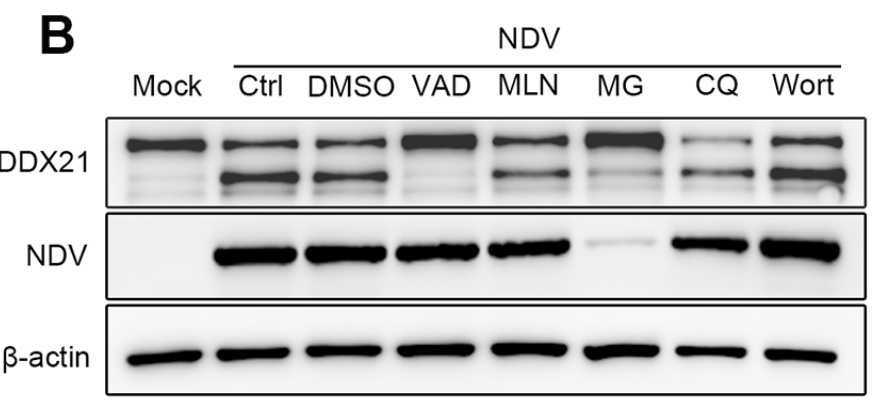

D

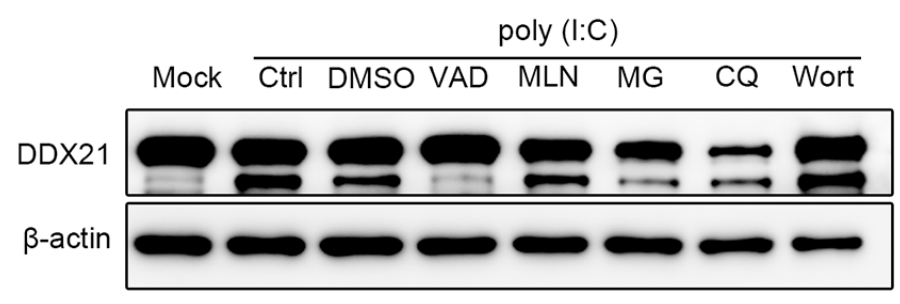


A

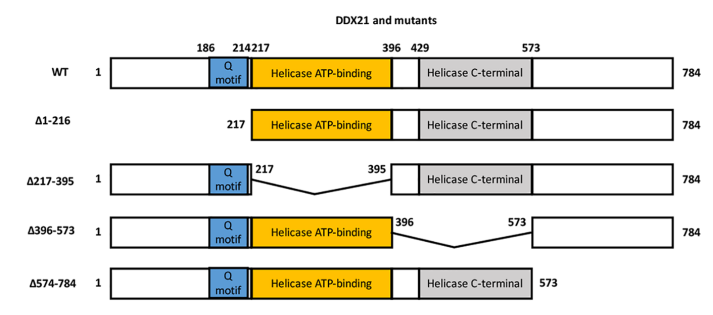

D
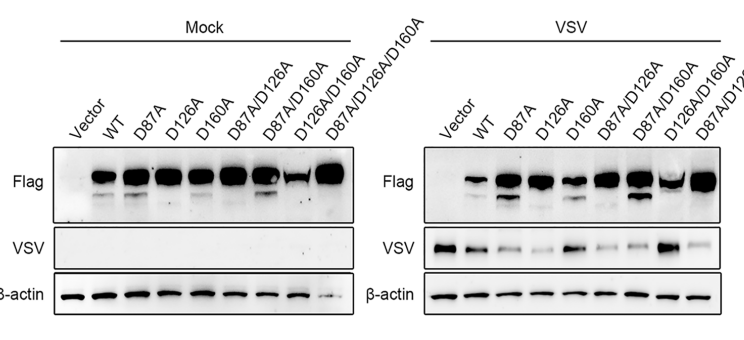

G

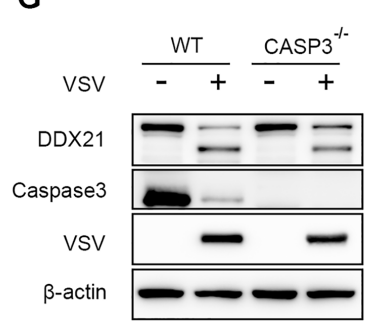

B

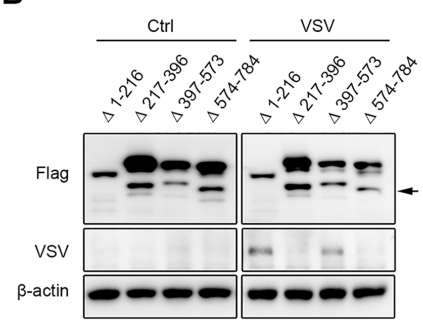

E

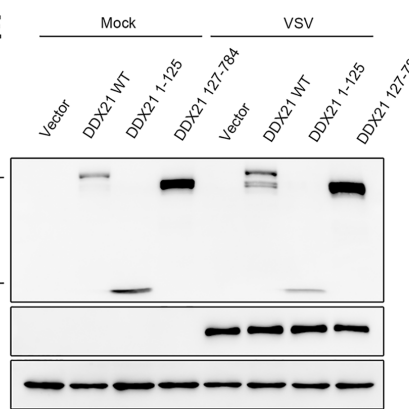

C

\section{Caspase cleavage prediction for Q9NR30 :}

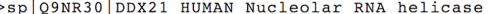

MPGKLRSDAGLESDTAMKKGETLRKQTEEKEKKEKPKSDKTEEIAEEEETVFPKAKQVKK 60 AEPSEVDMNSPKSKKAKKKEEPSQNDISPRTKSLRKKKEPIEKKVVSSKTKKVTKNEEP 120 VEQKEGAFSNFPISEETIKLLKGRGVTFLFPIQAKTF FHVYYSGKDLIAQARTGTGKTFSF 240 AIP

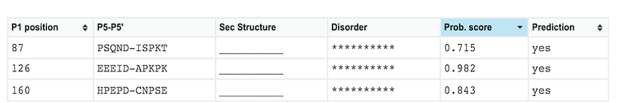



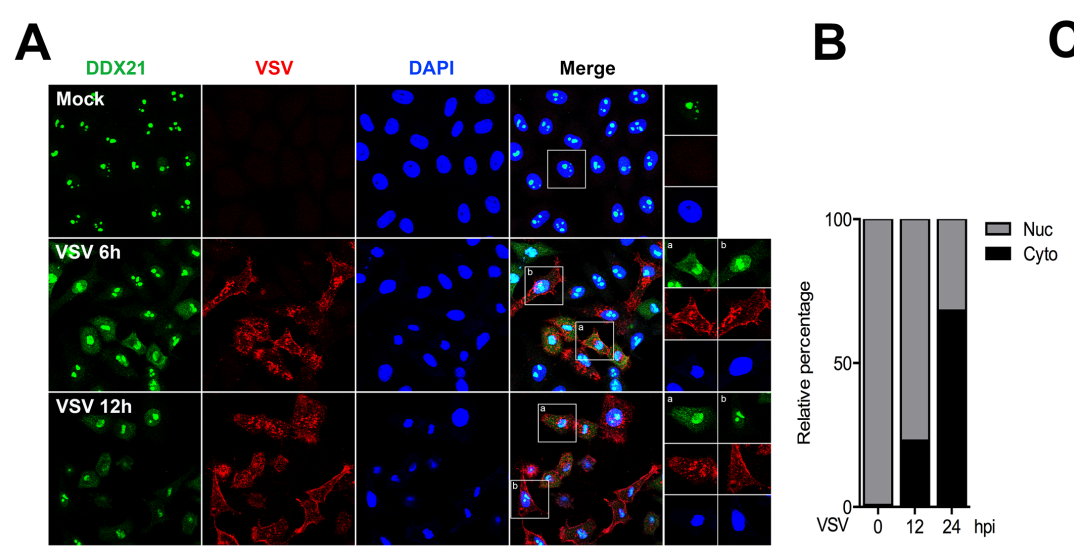

E

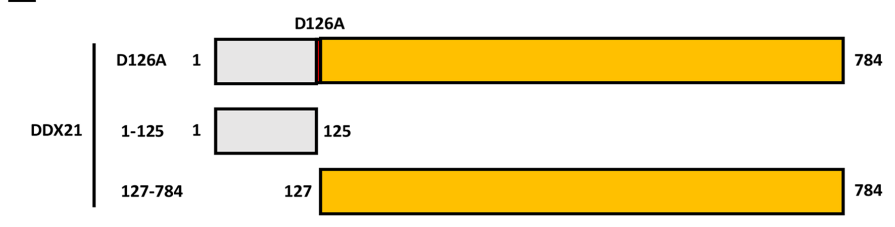

F

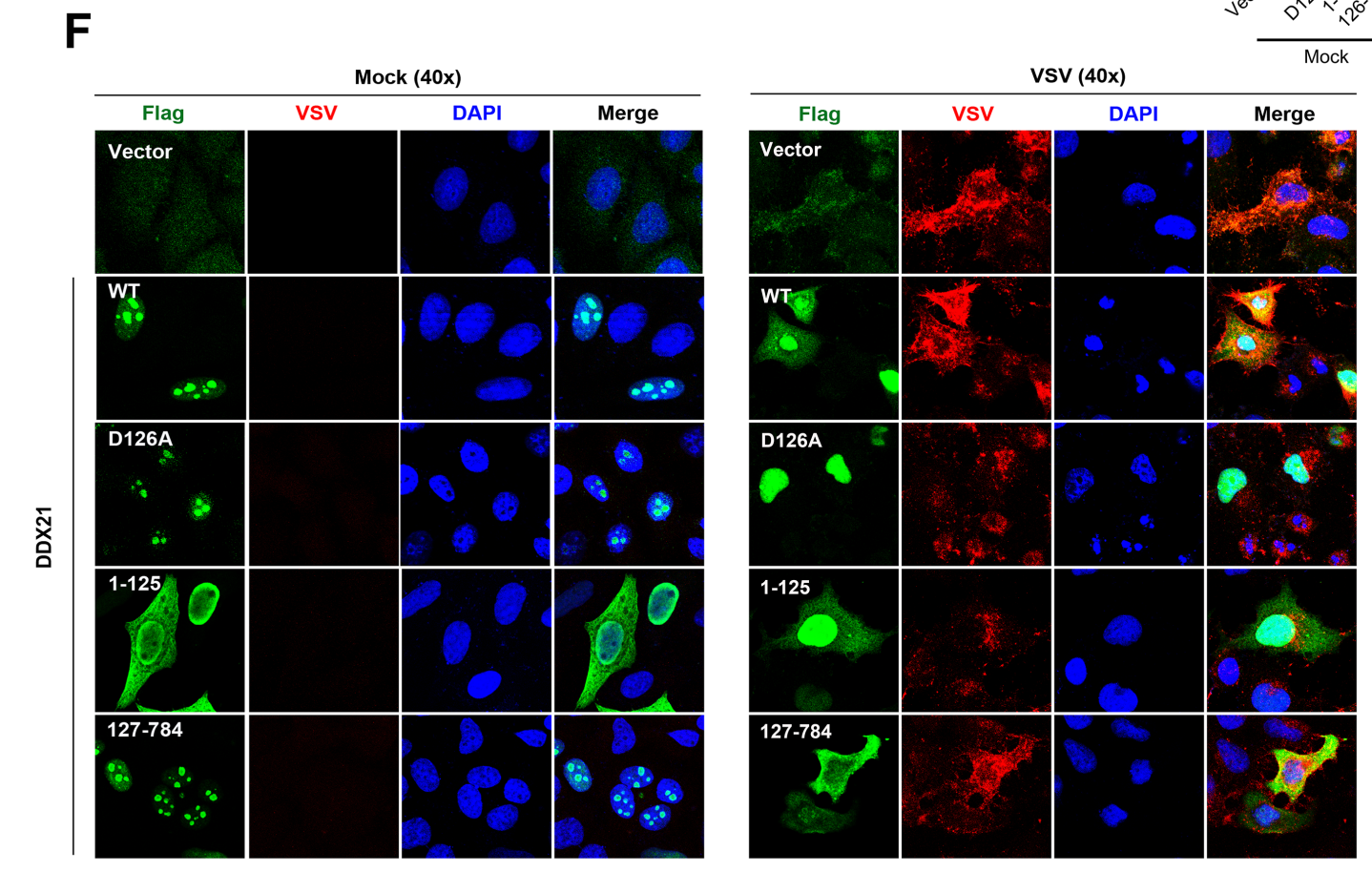

D
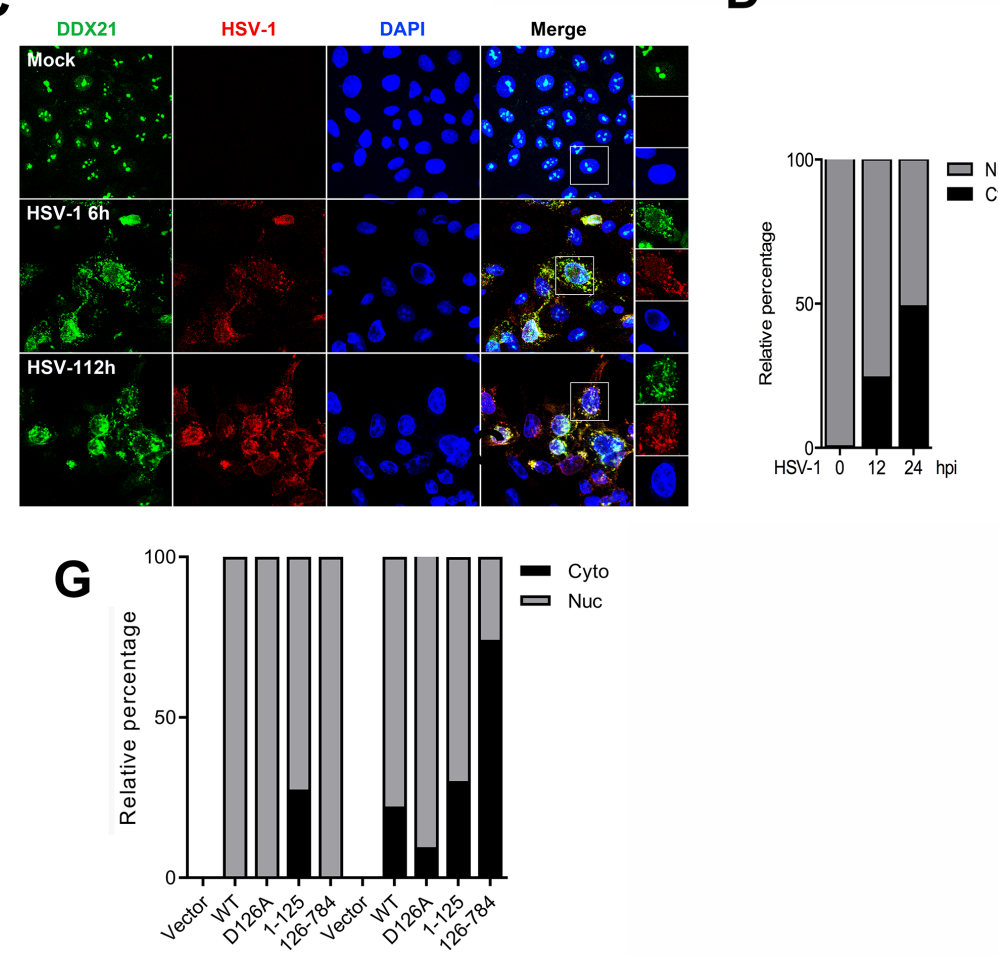

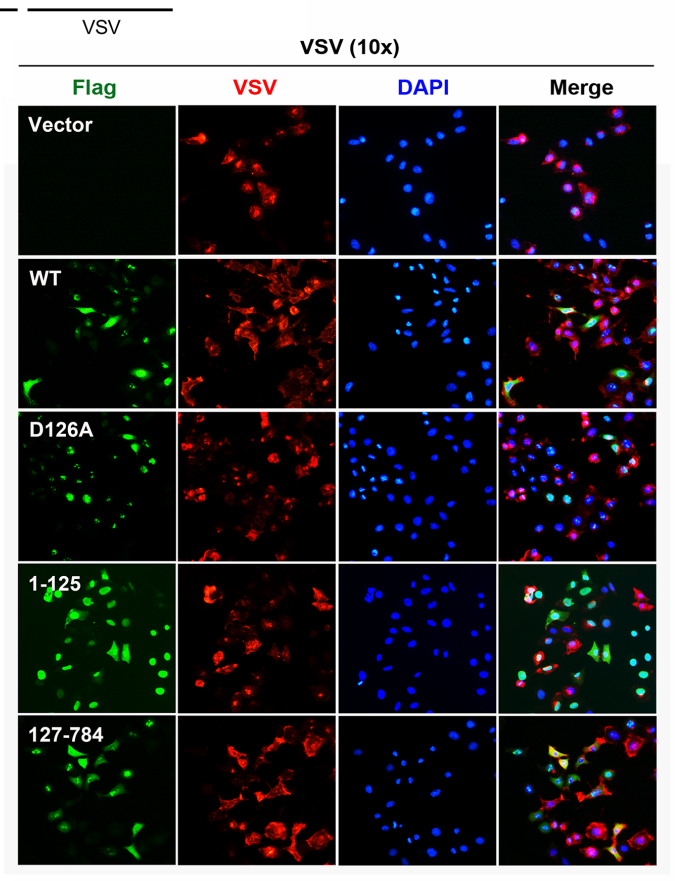


A

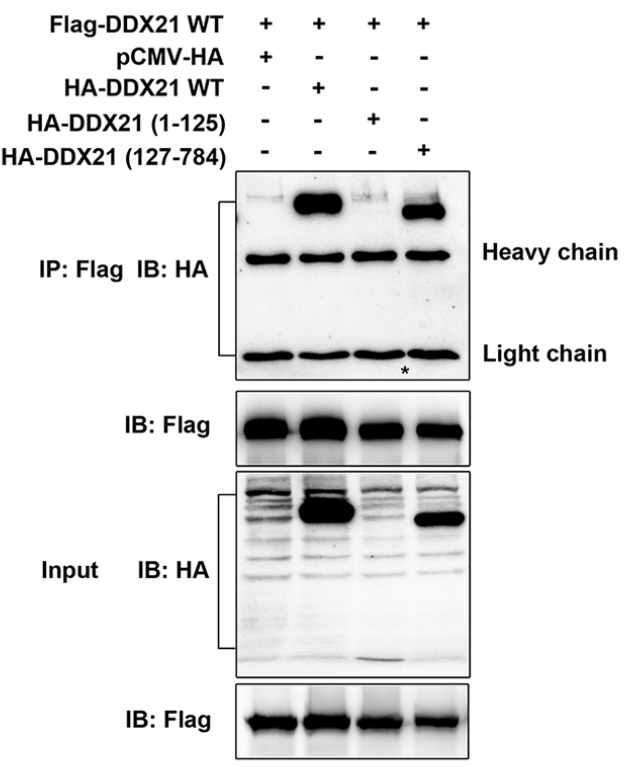

D

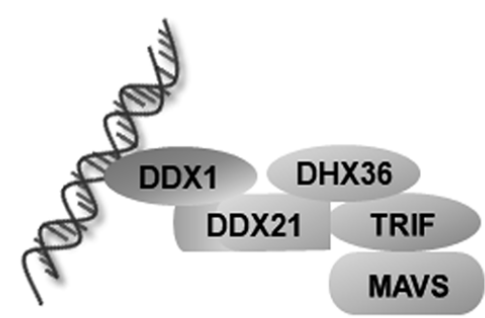

E
B
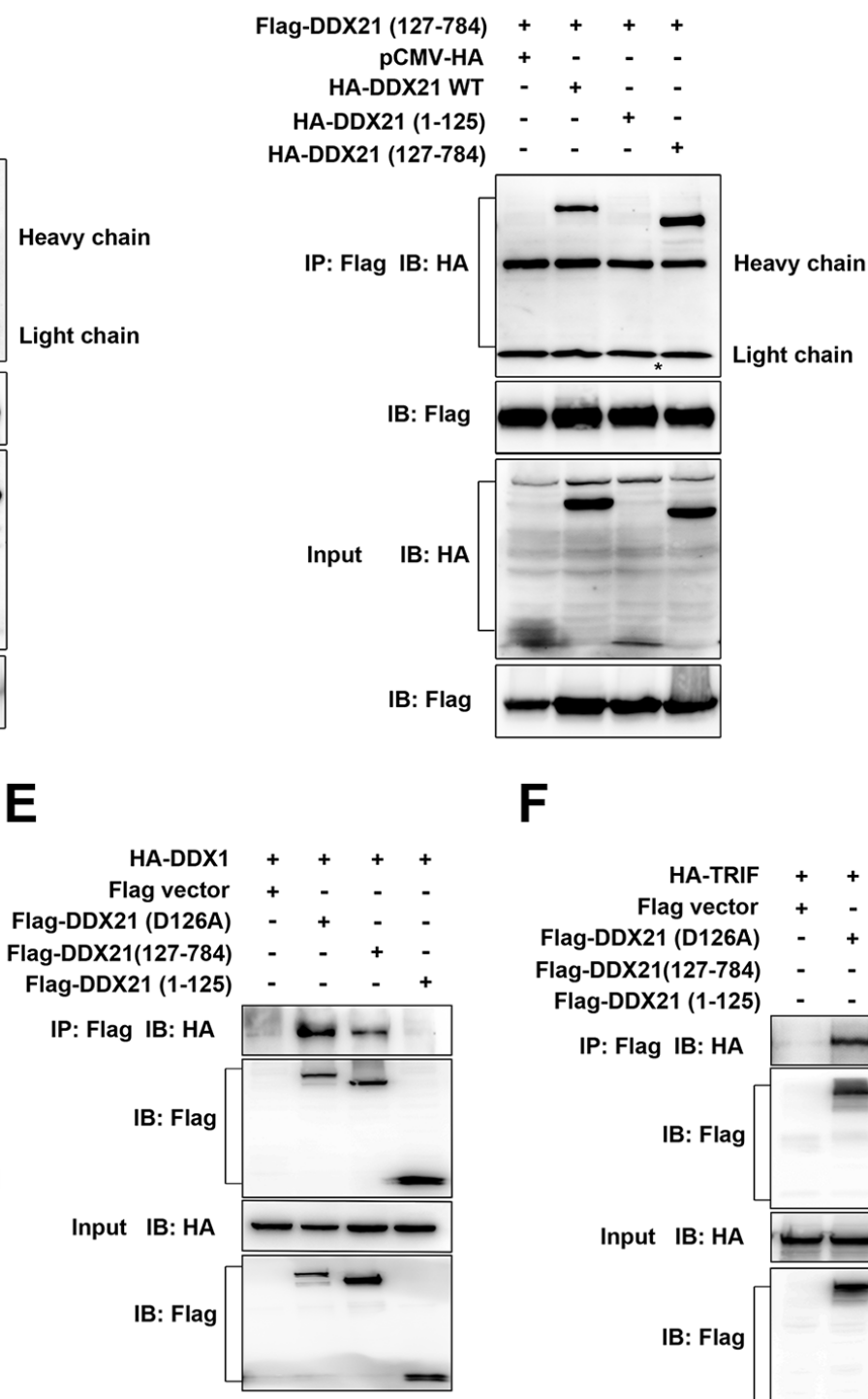

C
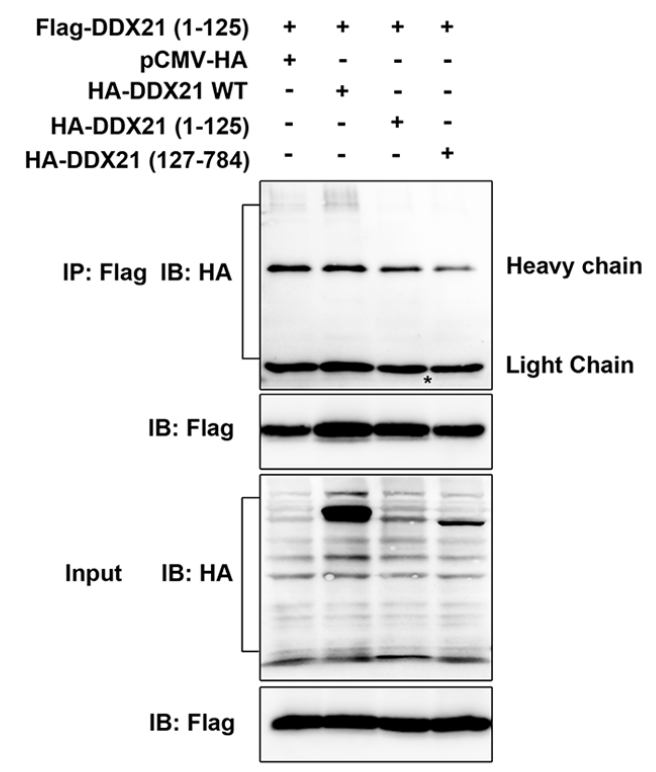

G
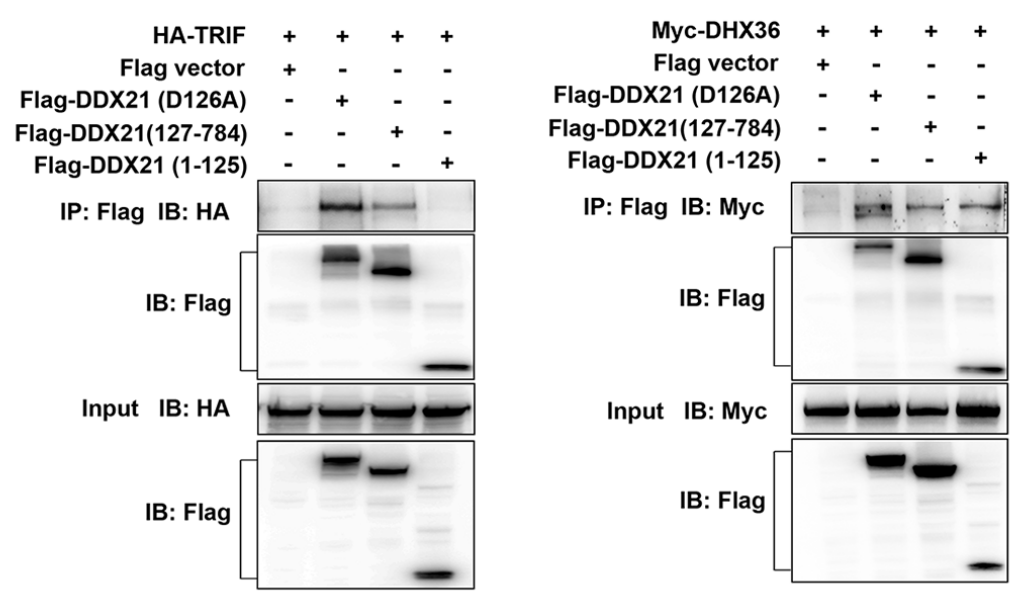


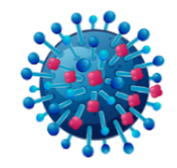

Early infection

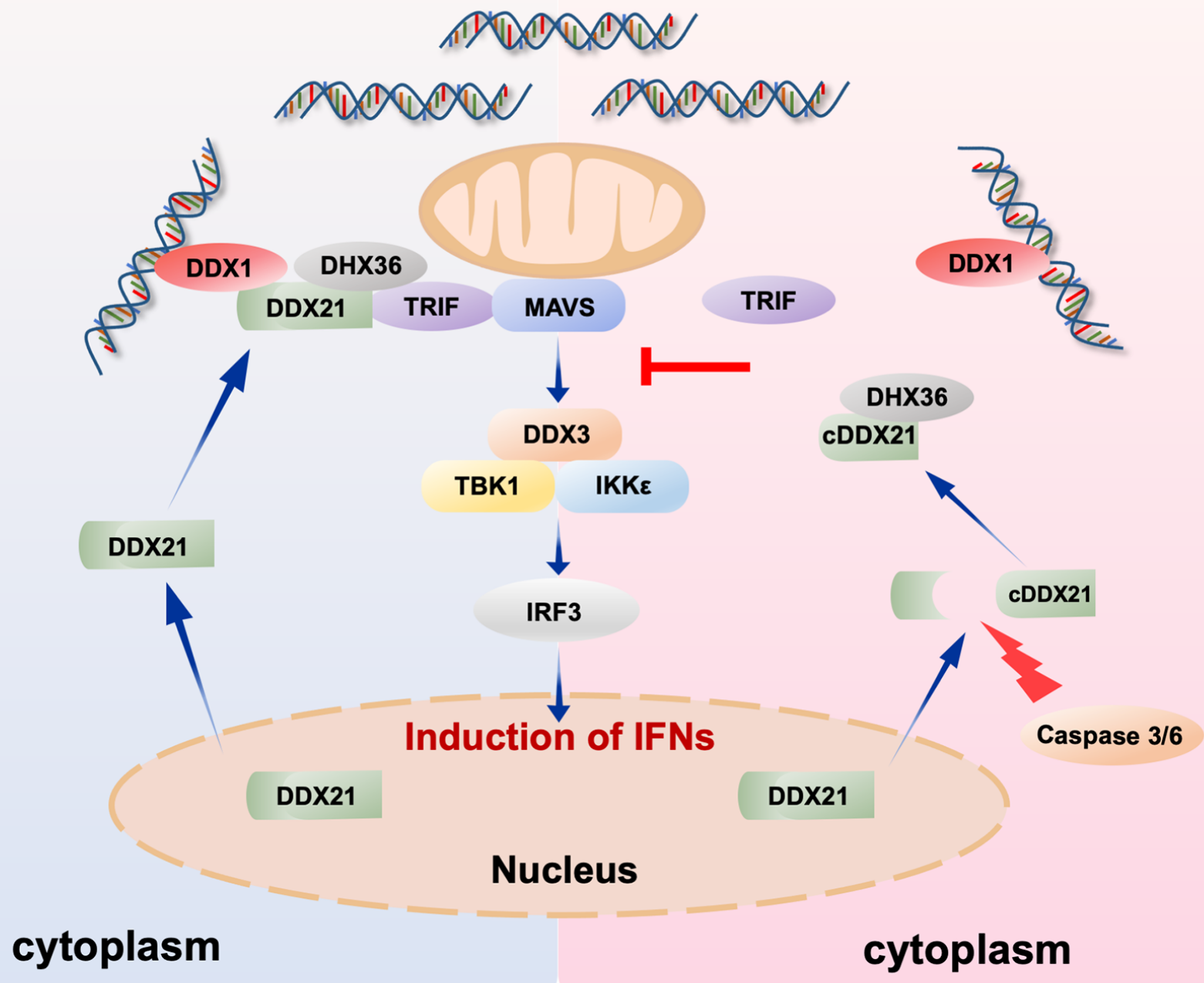

